\title{
2D facial landmark localization method for multi-view face synthesis image using a two-pathway generative adversarial network approach
}

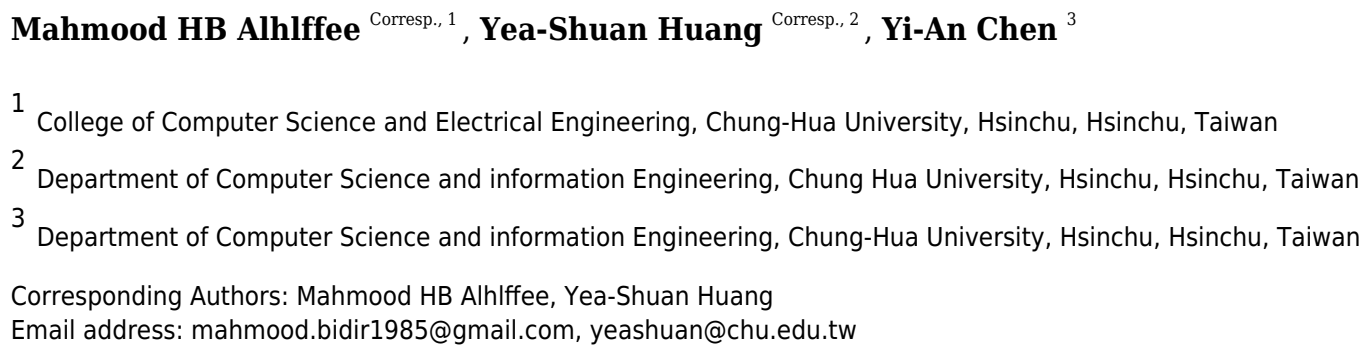

One of the key challenges in facial recognition is multi-view face synthesis from a single face image. The existing generative adversarial network (GAN) deep learning methods have been proven to be effective in performing facial recognition with a set of preprocessing, post-processing and feature representation techniques to bring a frontal view into the same position in-order to achieve highaccuracy face identification. However, these methods still perform relatively weak in generating high quality frontal-face image samples under extreme face pose scenarios. The novel framework architecture of the twopathway generative adversarial network (TP-GAN), has made commendable progress in the face synthesis model, making it possible to perceive global structure and local details in an unsupervised manner. More importantly, the TP - GAN solves the problems of photorealistic frontal view synthesis by relying on texture details of the landmark detection and synthesis function, which limits its ability to achieve the desired performance in generating high-quality frontal face image samples under extreme pose. We propose, in this paper, a landmark feature-based method (LFM) for robust pose-invariant facial recognition, which aims to improve image resolution quality of the generated frontal faces under a variety of facial poses. We therefore augment the existing TP-GAN generative global pathway with a well-constructed 2D face landmark localization to cooperate with the local pathway structure in a landmark sharing manner to incorporate empirical face pose into the learning process, and improve the encoder-decoder global pathway structure for better representation of facial image features by establishing robust feature extractors that select meaningful features that ease the operational workflow toward achieving a balanced learning strategy, thus significantly improving the photorealistic face image resolution. We verify the effectiveness of our proposed method on both Multi-PIE and FEI datasets. The quantitative and qualitative experimental results show that our proposed 
method not only generates high quality perceptual images under extreme poses but also significantly improves upon the TP-GAN results. 
1 Manuscript Title 2D facial landmark localization method for

2 multi-view face synthesis image using a two-pathway

3 generative adversarial network approach

4

5

6

7

8

Mahmood HB Alhlffee ${ }^{1}$, Yea-Shuan Huang ${ }^{2}$, Yi-An Chen ${ }^{3}$ 7

${ }^{1}$ College of Computer Science and Electrical Engineering, Chung-Hua University, Hsinchu, Hsinchu, Taiwan

2 Department of Computer Science and information Engineering, Chung Hua University, Hsinchu, Hsinchu, Taiwan

${ }^{3}$ Department of Computer Science and information Engineering, Chung Hua University, Hsinchu, Hsinchu, Taiwan

Corresponding Author:

Mahmood HB Alhlffee ${ }^{1}$,Yea-Shuan Huang ${ }^{2}$

${ }^{1}$ College of Computer Science and information Engineering, Chung Hua University, Hsinchu, Hsinchu, Taiwan

2 Department of Computer Science and information Engineering, Chung Hua University, Hsinchu, Hsinchu, Taiwan

Email address ${ }^{1}:$ mahmood.bidir1985@gmail.com

Email address ${ }^{2}$ : yeashuan@chu.edu.tw 


\section{Abstract}

One of the key challenges in facial recognition is multi-view face synthesis from a single face image. The existing generative adversarial network (GAN) deep learning methods have been proven to be effective in performing facial recognition with a set of pre-processing, postprocessing and feature representation techniques to bring a frontal view into the same position in-order to achieve high-accuracy face identification. However, these methods still perform relatively weak in generating high quality frontal-face image samples under extreme face pose scenarios. The novel framework architecture of the two-pathway generative adversarial network (TP-GAN), has made commendable progress in the face synthesis model, making it possible to perceive global structure and local details in an unsupervised manner. More importantly, the TP GAN solves the problems of photorealistic frontal view synthesis by relying on texture details of the landmark detection and synthesis function, which limits its ability to achieve the desired performance in generating high-quality frontal face image samples under extreme pose. We propose, in this paper, a landmark feature-based method (LFM) for robust pose-invariant facial recognition, which aims to improve image resolution quality of the generated frontal faces under a variety of facial poses. We therefore augment the existing TP-GAN generative global pathway with a well-constructed $2 \mathrm{D}$ face landmark localization to cooperate with the local pathway structure in a landmark sharing manner to incorporate empirical face pose into the learning process, and improve the encoder-decoder global pathway structure for better representation of facial image features by establishing robust feature extractors that select meaningful features that ease the operational workflow toward achieving a balanced learning strategy, thus significantly improving the photorealistic face image resolution. We verify the effectiveness of our proposed method on both Multi-PIE and FEI datasets. The quantitative and qualitative experimental results show that our proposed method not only generates high quality perceptual images under extreme poses but also significantly improves upon the TP-GAN results.

\section{Introduction}

Face recognition is one of the most commonly used biometric systems for identifying individuals and objects on digital media platforms. Due to changes in posture, illumination, and occlusion, face recognition faces multiple challenges. The challenge of posture changes comes into play when the entire face cannot be seen in an image. Normally, this situation may happen when a person is not facing the camera during surveillance and photo tagging. In order to overcome these difficulties, several promising face recognition algorithms based on deep learning have been developed, including generative adversarial networks (GANs). These methods have been shown to work more efficiently and accurately than humans at detection and recognition tasks. In such methods, pre-processing, post-processing, and multitask learning or feature representation techniques are combined to provide high accuracy results on a wide range of benchmark data sets (Junho et al., 2015; Chao et al., 2015; Xi et al., 2017; Jian et al., 2018). The main hurdle to these methods is multi-view face synthesis from a single face image (Bassel, Ilya \& Yuri, 2021; Chenxu et al., 2021; Yi et al., 2021; Hang et al., 2020; Luan, Xi \& Xiaoming, 2018; Rui et al., 
67 2017). Furthermore, a recent study (Soumyadip et al., 2016) emphasized that compared with

68 frontal face images with yaw variation less than 10 degrees, the accuracy of recognizing face 69 images with yaw variation more than 60 degrees is reduced by $10 \%$. The results indicate that 70 pose variation continues to be a challenge for many real-world facial recognition applications.

71 The existing approaches to these challenges can be divided into two main groups. In a first

72 approach, frontalization of the input image is used to synthesize frontal-view faces (Meina et al., 73 2014; Tal et al., 2015; Christos et al., 2015; Junho et al., 2015), meaning that traditional facial

74 recognition methods are applicable. Meanwhile, the second approach focuses on learning

75 discriminative representations directly from non-frontal faces through either a one-joint model or

76 multiple pose-specific models (Omkar, Andrea \& Andrew, 2015; Florian, Dmitry \& James,

77 2015). It is necessary to explore the above approaches in more detail before proceeding. For the

78 first approach, the conventional approaches often make use of robust local descriptors, (such as

79 John, 1985; Lowe, 1999; Ahonen, Hadid \& Pietikäinen, 2006; Dalal \& Triggs, 2015), to account

80 for local distortions and then adapt the metric learning method to achieve pose invariance.

81 Moreover, local descriptors are often used (Kilian \& Lawrence, 2009; Tsai-Wen et al., 2013)

82 approaches to eliminate distortions locally, followed by a metric learning method to prove pose

83 invariance. However, due to the tradeoff between invariance and discriminability, this type of

84 approach is relatively weak in handling images with extreme poses. A second approach, often

85 known as face rotation, uses one-joint models or multiple pose-specific models to learn

86 discriminative representations directly from non-frontal faces. These methods have shown good

87 results for near-frontal face images, but they typically perform poorly for profile face images

88 because of severe texture loss and artifacts. Due to this poor performance, researchers have been

89 working to find more effective methods to reconstruct positive facial images from data (Yaniv et

90 al., 2014; Amin \& Xiaoming, 2017; Xi et al., 2017). For instance, (Junho et al., 2015), adopted a

91 multi-task model to improves identity preservation over a single task model from paired training

92 data. Later on (Luan, Xi \& Xiaoming, 2017; Rui et al., 2017), their main contribution was a

93 novel two-pathway GAN architecture tasks for photorealistic and identity preserving frontal

94 view synthesis starting from a single face image. Recent work by (Bassel, Ilya \& Yuri, 2021;

95 Chenxu et al., 2021; Yi et al.,2021) has demonstrated advances in the field of face recognition.

96 During pose face transformation, however, some of the synthetic faces appeared incomplete and

97 lacked fine detail. So far, the TP-GAN (Rui et al., 2017) has made significant progress in the

98 face synthesis model, which can perceive global structure and local details simultaneously in an

99 unsupervised manner. More importantly, TP-GAN solves the photorealistic frontal view

100 synthesis problems by collecting more details on local features for a global encoder-decoder

101 network along with synthesis functions to learn multi-view face synthesis from a single face

102 image. However, we argue that TP-GAN has two major limitations. First, it is critically

103 dependent on texture details of the landmark detection. To be more specific, this method focuses

104 on the inference of the global structure and the transformation of the local texture details, as their

105 corresponding feature maps, to produce the final synthesis. The image visual quality results

106 indicate that these techniques alone have the following deficiencies: A color bias can be 
107

108

109

110

111

112

113

114

115

116

117

118

119

120

121

122

123

124

125

126

127

128

129

130

131

132

133

134

135

136

137

138

139

140

141

142

143

144

145

146

observed between the synthetic frontal face obtained by TP-GAN method and the input corresponding to non-frontal input. In some cases, the synthetic faces are even incomplete and fall short in terms of fine detail. Therefore, the quality of the synthesized images still cannot meet the requirements for performing specific facial analysis tasks, such as facial recognition and face verification. Second, it uses a global structure, four local network architectures and synthesis functions for face frontalization, where training and inference are unstable under large data distribution, which makes it ineffective for synthesising arbitrary poses. The goal of this paper is to address these challenges through a landmark feature-based method (LFM) for robust pose-invariant facial recognition to improve image resolution under extreme facial poses.

In this paper, we make the following contributions:

The LFM is a newly introduced method for the existing generative global pathway structure that utilizes a 2D face landmark localization to cooperate with the local pathway structure in a landmark sharing manner to incorporate empirical face pose into the learning process. LFM of target facial details provides guidance to arbitrary pose synthesis, whereas the four-local patch network architecture remains unchanged to capture the input facial local perception information. The LFM provides an easy way for transforming and fitting two-dimensional face models in order to achieve target pose variation and learn face synthesis information from generated images.

In order to better represent facial image features, we use a denoising autoencoder (DAE) to modify the structure of the generator's global-path encoder and decoder. The goal of this modification is to train the encoder decoder with multiple noise levels so that it can learn about the missing texture face details. Adding noise to the image pixels causes them to diffuse away from the manifold. As we apply DAE to the diffused image pixels, it attempts to pull the data points back onto the manifold. This implies that DAE implicitly learns the statistical structure of the data by learning a vector field from locations with no data points back to the data manifold. As a result, encoder-decoders must infer missing pieces and retrieve the denoised version in order to achieve balanced learning behavior.

We optimize the training process using an accurate parameter configuration for a complex distribution of facial image data. By re-configuring the parameters (such as the learning rate, batch size, number of epochs, and etc.), the GAN performance can be better optimized during the training process. Occasionally, unstable "un-optimized" training for the synthetic image problem results in unreliable images for extreme facial positions.

\section{Related Work}

In this section, we focus on the most recent studies which are related to the multi-view face synthesis problem using deep learning approaches. The deep learning approaches including face normalization, generative adversarial network and facial landmark detection, are reviewed.

\section{Face Normalization}


147 Face normalization, or multi-view face synthesis from a single face image, is a unique challenge

148 for computer vision systems due to its ill-posed problem. The existing solutions to address this 149 challenge can be classified into three categories: 2D/3D local texture warping methods (Tal et 150 al., 2015; Xiangyu et al., 2015), statistical methods (Christos et al., 2015; Li et al., 2014), and 151 deep learning methods (Xi et al., 2017; Luan, Xi \& Xiaoming, 2017). (Tal et al., 2015), 152 employed a single 3D reference surface for all query faces in order to produce face 153 frontalization. (Xiangyu et al., 2015), employed a pose and expression normalization method to

154

155

156

157

158

159

160

161

162

163

164

165

166

167

168

169

170

171

172

173

174

175

176

177

178

179

180

181

182

183

184

185

186 recover the canonical-view. (Christos et al., 2015), proposed a joint frontal view synthesis and landmark localization method. (Li et al., 2014), the authors concentrated on local binary patternlike feature extraction. (Xi et al., 2017), proposed a novel deep 3DMM-conditioned face frontalization GAN in order to achieve identity-preserving frontalization and high-quality images by using a single input image with a $90^{\circ}$ face pose. (Luan, Xi \& Xiaoming, 2017), proposed a single-pathway framework called the disentangled representation learning-generative adversarial network (DR-GAN) to learn identity features that are invariant to viewpoints, etc.

\section{Generative Adversarial Networks (GANs)}

The GAN is one of the most interesting research frameworks that is used for deep generative models proposed by (Ian et al., 2014). The theory behind the GAN framework can be seen as a two-player non-cooperative game to improve the learning model. A GAN model has two main components, generator (G) and discriminator (D). G generates a set of images that is as plausible as possible in order to confuse the $\mathrm{D}$, while the $\mathrm{D}$ works to distinguish the real generated images from the fake. The convergence is achieved by alternately training them. The main difference between GANs and traditional generative models is that GANs generate whole images rather than pixel by pixel. In a GAN framework, the generator consists of two dense layers and a dropout layer. A normal distribution is used to sample the noise vectors and feed them into the generator networks. The discriminator can be any supervised learning model. GANs have been proven effective for a wide range of applications, such as image synthesis (Rui et al., 2017; Yu et al., 2018; Yu et al., 2020), image super-resolution (Christian et al., 2017), image-to-image translation (Jun-Yan et al., 2017), etc. Several effective GAN models have been proposed to cope with the most complex unconstrained face image situations, such as changes in pose, lighting and expression. For instance, (Alec, Luke \& Soumith, 2016), proposed a deep convolutional GAN to integrate a convolutional network into the GAN model to achieve more realistic face image generation. (Mehdi \& Simon, 2014), proposed a conditional version of the generative adversarial net framework in both generator and discriminator. (Augustus, Christopher \& Jonathon, 2017), presented an improved version of the Cycle-GAN model called "pixel2pixel" to handle the image-to-image translation problems by using labels to train the generator and discriminator. (David, Thomas \& Luke, 2017), proposed a boundary equilibrium generative adversarial network (BE-GAN) method, which focuses on the image generation task to produce high-quality image resolution, etc. 
187 Facial Landmark Detection

188 The face landmark detection algorithm is one of most successful and fundamental components in 189 a variety of face applications, such as object detection and facial recognition. The methods used 190 for facial landmark detection can be divided into three major groups; holistic methods, 191 constrained local methods, and regression-based methods. In the past decade, deep learning 192 models have proven to be a highly effective way to improve landmark detection. Several existing

193

194

195

196

197

198

199

200

201

202

203

204

205

206

207

208

209

210

211

212

213

214

215

216

217

218

219

220

221

222 methods are considered to be good baseline solutions to the 2D face alignment problem for faces with controlled pose variation (Xuehan \& Fernando, 2013; Georgios, 2015; Xiangyu et al., 2016; Adrian \& Georgios, 2017). (Xuehan \& Fernando, 2013), proposed supervised descent method, which learns the general descent directions in a supervised manner. (Georgios, 2015), in their method, a sequence of Jacobian matrices and hessian matrices is determined by using regression. (Xiangyu et al., 2016), proposed a 3D model with cascaded convolutional neural network to solve the self-occlusion problem. (Adrian \& Georgios, 2017), proposed a guided-by-2D landmarks convolutional neural network that converts 2D annotations into 3D annotations, etc.

We can summarize some important points from our related work. Despite the fact that the existed methods produced good results on the specific face image datasets for which they were designed and provided robust alignment across poses, they are difficult to replicate if they are applied alone to different datasets. This is especially true for tasks like facial normalization or other face synthesis tasks, where deep structure learning methods still fail to generate highquality image samples under extreme pose scenarios, which results in significantly inferior final results.

\section{Proposed Method}

In this section, we shall first briefly describe the existing TP-GAN architecture and then describe our proposed LFM method in detail.

\section{TP-GAN Architecture}

Based on the structure shown in Fig 1, the TP-GAN framework architecture consists of two stages. The first stage is a generator of two-pathways $\mathrm{CNN} G_{\theta_{G}}$ that is parameterized by $\theta_{G}$. Each pathway has encoder-decoder $\left\{G_{\theta_{E}} G_{\theta_{D}}\right\}$ structure and combination of loss functions, a local pathway $\left\{G_{\theta_{E}^{l}}, G_{\theta_{D}^{l}}\right\}$ of four landmark patch networks $G_{\theta_{i}^{l}}, i \in\{0,1,2,3\}$ to capture the local texture around four facial landmarks, and one global network $\left\{G_{\theta_{E}{ }^{g}} G_{\theta_{D}}\right\}$ to process the global face structure. Furthermore, the bottleneck layer $\left(G_{g}\right)$, which is the output of $G_{\theta_{E}}$, is typically used for classification tasks with the cross-entropy loss $L_{\text {cross - entropy. }}$ A global pathway helps to integrate facial features with their long-range dependencies and, therefore, to create faces that preserve identities, especially in cases of faces with large pose angles. In this way, we can learn a 
223

224

225

226

227

228

229

230

231

232

233

234

235

236

237

238

239

240

241

242

243

244

245

246

247

248

249

250

251

252

253

254

255

256

257

258

259

260

richer feature representation and generate inferences that incorporate both contextual dependencies and local consistency. The loss functions, including pixel-wise loss, symmetry loss, adversarial loss, and identity preserving loss, are used to guide an identity preserving inference of frontal view synthesis. The discriminator $D_{\theta_{D}}$ is used to distinguish real facial images $I^{F}$ or 'ground-truth $(G T)$ frontal view' from synthesized frontal face images $G_{\theta_{G}}\left(I^{P}\right)$ or 'synthesized-frontal $(S F)$ view'. A second stage involves a light-CNN model that is used to compute face dataset's identity-preserving properties. For a more detailed description, see (Rui et al., 2017).

\section{LFM for Generative Global Pathway Structure}

To the best of our knowledge, this is the first study to integrate an LFM with the existing TPGAN global pathway structure for training and evaluation purposes. In this work, we exploit a landmark detection mechanism (Adrian \& Georgios, 2017) that proposed for 2D-to-3D facial landmark localization to help our model obtain a high quality frontal-face image resolution. Face landmarks are the most compressed representation of a face that maintains information such as pose and facial structure. There are many situations where landmarks can provide advanced facerelated analyses without using whole face images. The landmark method used in this study was explored at (Xi Y et al., 2017; Shuicheng \& Jiashi. 2018; Xing D, Vishwanath. Sindagi \& Vishal. 2018). These methods can achieve high accuracy of face alignment by cascaded regression methods. Methods like these work well when particular poses are chosen without taking other factors into consideration, such as facial characteristics. We found that facial characteristics can play an imperative role in improving the results of the current state of the art. By adding landmarks to augment the synthesized faces, recognition accuracy will be improved since these landmarks rely on generative models to enhance the information contained within them. The process for generating facial images is shown in Fig 1. We will discuss our Fig 2 architecture in the subsequent paragraph. We perform a face detection to locate the face in the Multi-PIE and FEI datasets. The face detection can be achieved by using a Multi-Task Cascade CNN through the MTCNN library (Kaipeng Z et al.,2016). After that, cropping and processing of the profile image. A local pathway of four landmark patch networks $G_{\theta_{i}^{l}}, i \in\{0,1,2,3\}$ to capture the local

texture around four facial landmarks. Each patch learns a set of filters for rotating the centercropped patch (after rotation, the facial landmarks remain in the center) to its corresponding frontal view. Then, we used a multiple feature map to combine the four facial tensors into one. Each tensor feature is placed at a "template landmark location" and a max-out fusing strategy is used to ensure that stitches on overlapping areas are minimized. Then, a 2D zero padding technique is used to fill out the rows and columns around the template landmark location with zeros. Nevertheless, local landmark detection alone cannot provide accurate texture detail for a face that has a different shape, or multiple views, because all generated local synthesis faces have the same fixed patch (or template) centralized location, regardless of their shape

Peer] Comput. Sci. reviewing PDF | (CS-2021:10:66723:2:0:NEW 25 Jan 2022) 
261

262

263

264

265

266

267

268

269

270

271

272

273

274

275

276

277

278

279

280

281

282

283

284

285

286

287

288

289

290

291

292

293

294

295

296

297

298

characteristics. The challenge becomes even greater with these shapes when the face is under extreme poses. A texture can be defined as a function of the spatial variation of brightness intensity of pixels in an image. Each texture level represents a variable, with variations such as smoothness, coarseness, regularity, and etc., of each surface oriented in different directions. Our work focuses on two important phenomena: rotation and noise "noise is a term used to describe image information that varies randomly in brightness or color". As a result, if the methods used to eliminate these common phenomena are unreliable, the results will be less accurate; therefore, in practice, the methods used to create the images should be as robust and stable as possible. In addition, the images may differ in position, viewpoint, and light intensity, all of which can influence the final results, challenging texture detail capturing. In order to overcome these challenges, we must adapt a method that can capture and restore the missing texture information. The key to solving this problem is a landmark localization method based on regression. Our work utilized Face Alignment Network (FAN). A FAN framework is based on the HourGlass (HG) architecture, which integrates four Hourglass models to model human pose through hierarchical, parallel, and multi-scale integration to improve texture maps by reconstructing selfoccluded parts of faces. The landmark detection algorithm captures and restores the texture details of the synthesised face image by repositioning the appearance spot of the mismatched or drifted patch "template landmark location". Figure 3 illustrates some examples. Our method allows us to treat faces that have a variety of shape characteristics. In this way, the spatial variations, smoothness, and coarseness that arise due to mismatched or drifted pixels between local and global synthesized faces are eliminated. Typical landmark templates are approximately the same size as a local patch network, but each region has its own structure, texture, and filter. Next, we combined the local synthesis image with the global synthesis image (or two textures) for data augmentation. Every patch of our FAN has its own augmented channels, and each patch has its own RGB along with a depth map (D) input for each 2D local synthesis image. In this way, the texture details help us to build a more robust model around the face patch region and enhance generalization. Even though landmark feature extraction may result in some incongruous or over-smoothing due to noise, it still remains an important method for incorporating pose information during learning.

The landmark detection algorithm for our synthesis face image was built using 68 points. We then reconstructed those synthesis image into four uniform patches (or templates), Leye $=G_{\theta_{0}^{g}}$ ,$i \in 0$, Reye $=G_{\theta_{1} g^{,}}, i \in 1$, Nose $=G_{\theta_{2}^{g}}, i \in 2$ and Mouth $=G_{\theta_{3}}, i \in 3$, and each patch is comprised of convolutional components. Each patch region has its own filter, which contains different texture details, regain size and structure information. Individual filters provide more details about specific areas in an image, such as pixels or small areas with a high contrast or that are different in color or intensity from the surrounding pixels or areas. Then, one layer of convolutional neural networks is used to coordinate and extract intermediate features. For our method to work more effectively, we remove layers 5, 6, and 7 from ConvNet $\left\{G_{\theta_{D}^{g}}\right\}$ and replace them after the

Peer] Comput. Sci. reviewing PDF | (CS-2021:10:66723:2:0:NEW 25 Jan 2022) 
299

300

301

302

303

304

305

306

307

308

309

310

311

312

313

314

315

316

317

318

319

320

321

322

323

324

325

326

327

328

329

330

331

332

333

334

335

336

337

concatenation stage. Those layers' act as a visualization feature map for a specific input of a fontal-face image in order to increase the amount of visual information kept by subsampling layers' structure. Then, we merged the features tensors of the local and global pathways into one tensor to produce the final synthesis face. Table 1 shows the workflow of all operational steps. The landmark method provides useful information for large-pose regions, e.g., $90^{\circ}$, which helps our model to produce more realistic images.

\section{Global Pathway Encoder-Decoder Structure}

In this section we describe our encoder-decoder formulation. Inspired by the work of (Jimei Y et al., 2016), for the DAE, our aim is to train the encoder-decoder with multiple noise levels in order to learn more about the missing texture face details of the input face image and preserve the identity of the frontal-view image $I^{F}$ from the profile image $I^{P}$. The encoder-decoder mechanism has to discover and capture information between the dimensions of the input in order to infer missing pieces and recover the denoised version. In a subsequent paragraph, we will discuss encoder-decoders in more technical details. The idea starts with assuming that the input data points (image pixels) lies on a manifold in $\mathbb{R}^{N}$. Adding noise to the data image pixel results in diffusing away from the manifold. When we apply DAE to the diffused data image pixels, it tries to pull the data point back onto the manifold. Therefore, DAE learns a vector field pointing from locations with no data point back to the data manifold, implying that it implicitly learns the statistical structure of the data. However, a sparse coding model has been shown to be a good model for image denoising. We assume that group sparse coding, which generalizes standard sparse coding, is effective for image denoising as well, and we will view from a DAE perspective. The encoding function of sparse coding occurs in the inference process, where the network infers the latent variable $s$ from noisy input $\hat{x}$. Each individual symbol is defined here. Let $f_{e}$ be the RGB components of the input face image, $\Phi$ is the method that splits the input image into its RBG components, $\Lambda$ is a set of weights and bias for the DAE, and $a$ is the activation function. In our case, the iterative shrinkage-thresholding algorithm (ISTA) is used to perform inference and is formulated as follows:

$$
s=f_{e}(\tilde{x} ;\{\Phi, \wedge, a\})=\operatorname{ISTA}(\tilde{x} ; \Phi, \wedge, a)
$$

The decoding function is the network's reconstruction of the input from the latent variable.

$$
\hat{x}=f_{d}(s ; \Phi)=\Phi_{s}
$$

where $f_{d}$ is a denoising function.

The DAE method can be used to learn $\wedge$ through the following. For each input data point as shown in Fig 4, we construct a noisy input by adding Gaussian white noise (GWN) to the original input profile images, as given in: $\tilde{x}=x+v$, where $v \sim N\left(O, \sigma^{2} I\right)$. Here, $I$ is an $N \times N$ 
338 identity matrix, where $N$ is the size of input data (batch output of the generator), and $\sigma^{2}$ is the 339 noise variance which is the same in all directions. We use group sparse coding to denoise $\tilde{x}$, as

340

341

342

343

344

345

346

347

348

349

350

351

352

353

354

355

356

357

358

359

360

361

362

363

364

365

366

367

368

369

370

371 described in Equation (1) and Equation (2). The DAE is formulated as follows:

$$
E_{D A E}=\|x-\hat{x}\|_{2}^{2}
$$

We define DAE's reconstruction error as a square error between $x$ and $\hat{x}$.

Generally, the cost function can be another form of differentiable error measure, shown as follows:

$$
\Delta \wedge \propto-\partial E_{D A E} / \partial \wedge
$$

where $\partial E_{D A E} / \partial \wedge \rightarrow$ differentiate DAE's reconstruction error with respect to weights, and $\Delta \wedge$ is the change in weights, $\wedge$ can then be learned by doing gradient descent on the DAE's cost function.

Essentially, the $G_{\theta_{E}^{g}}$ encodes input data $x \in \mathbb{R}^{N}$ into a hidden representation: $h=f_{e}(x ; \theta) \in$ $\mathbb{R}^{M} . f_{e}$ is the encoder and $\theta$ is learning parameters of the DAE function. Then, it decodes $G_{\theta_{D}}$ the hidden representation into a reconstruction of the input data: $\hat{x}=f_{d}(h ; \theta)$. The objective for learning the parameter $\theta$ of an $\left\{G_{\theta_{E}^{g}}, G_{\theta_{D}^{g}}\right\}$ is to minimize the reconstruction error between $\hat{x}$ and $x$. Usually, there is some constraint on $\left\{G_{\theta_{E}^{g}}, G_{\theta_{D}}\right\}$ to prevent it from learning an identity transformation. For example, the dimension of $h$ is much smaller than the input data's dimension i.e. $M \ll N$, then $\left\{G_{\theta_{E}^{g}}, G_{\theta_{D}^{g}}\right\}$ will function similarly to principle component analysis (PCA). The hidden representation $\|h\|_{1}$ is small, then $G_{\theta_{E}^{g}}$ functions similarly to sparse coding. The DAE tries to remove noise from input data. Let $\tilde{x}=x+v$ be a noisy input by adding $v$ to an original input $x$. DAE takes $\tilde{x}$ as an input, then outputs a denoising signal $\hat{x}=f_{d}\left(f^{e}(\tilde{x} ; \theta) ; \theta\right)$. The objective function of a such technique is to minimize the error between $x$ and $\hat{x}$ by adjusting $\theta$, i.e., it tries to reconstruct the actual content well while not reconstructing noise. DAE can also be viewed as a generative model.

\section{Adversarial Networks}

Following (Ian et al., 2014) work, adversaries network consists of two components $(G)$ and $(D)$. "The loss function reflects the difference in distribution between the generated and original data". We will first review some technical aspects of the training process, and then the adversaries' network. In frontal view synthesis, the aim is to generate a photorealistic and 
372 identity-preserving frontal view image $\left(I^{F}\right)$ from a face image under a different pose, i.e. a 373 profile image $\left(I^{P}\right)$. During the training phase of such networks, pairs of corresponding $\left\{I^{F}, I^{P}\right\}$

374 from multiple identities $y$ are required. Input $I^{P}$ and output $I^{F}$ are both based on a pixel space of

375 size $W \times H \times C$ with a color channel $C$. We aim to learn a synthesis function that can output a

376 frontal view when given a profile image. This section will be omitted since it was already

377 explained in TP-GAN architecture. Optimizing the network parameters $\left(G_{\theta_{G}}\right)$ starts with

378 minimizing the specifically designed synthesis loss $\left(L_{\text {syn }}\right)$ and the aforementioned $L_{\text {cross - entropy }}$

379 . For a training set with $N$ training pairs of $\left\{I_{n}^{F}, I_{n}^{P}\right\}$, the optimization problem is expressed as 380 follows:

381

382

$$
\hat{\theta}_{G}=\frac{1}{N} \underset{\theta_{G}}{\operatorname{argmin}} \sum_{n=1}^{N}\left\{L_{\text {syn }}\left(G_{\theta_{G}}\left(I_{n}^{P}\right), I_{n}^{F}\right)+\alpha L_{\text {cross - entropy }}\left(G_{\theta_{E}^{g}}\left(I_{n}^{p}\right), y_{n}\right)\right\}
$$

383

384

385

386

387

388

389

390

391

392

393

394

395

396

397

398

399

400

401

402

403

404

405

where $\alpha$ is a weighting parameter and $L_{\text {syn }}$ is a weighted sum of individual losses that together constrain the image to reside within the desired manifold. Each individual loss function will be explained in the comprehensive loss functions section.

In order to generate the best images, we need a very good generator and discriminator. The reason for this is that if our generator is not good enough, we won't be able to fool the discriminator, resulting in no convergence. A bad discriminator will also classify images that make no sense as real, which means our model never trains, and we never produce the desired output. The image can be generated by sampling values from a Gaussian distribution and feeding them into the generator network. Based on a game-theoretical approach, our objective function is a minimax function.

$$
\min _{\theta_{G}} \max _{\theta_{D}}\left[\mathbb{E}_{I^{F} \sim P\left(I^{F}\right)} \log D_{\theta_{D}}\left(I^{F}\right)+\mathbb{E}_{I^{P} \sim P\left(I^{P}\right)} \log \left(1-D_{\theta_{D}}\left(G_{\theta_{G}}\left(I^{P}\right)\right)\right)\right]
$$

where $I^{F}$ presents as real frontal face images

$D_{\theta_{D}}$ presents as the discriminator

$G_{\theta_{G}}$ presents as the generator

$G_{\theta_{G}}\left(I^{P}\right)$ presents as synthesized frontal face images.

Using the discriminator to maximize the objective function allows us to perform gradient descent on it. The generator tries to minimize its objective function, so we can use gradient descent to compute it. In order to train the network, gradient ascent and descent must be alternated.

$$
\max _{\theta_{D}}\left[\mathbb{E}_{I^{F} \sim P\left(I^{F}\right)} \log D_{\theta_{D}}\left(I^{F}\right)+\mathbb{E}_{I^{P} \sim P\left(I^{P}\right)} \log \left(1-D_{\theta_{D}}\left(G_{\theta_{G}}\left(I^{P}\right)\right)\right)\right]
$$


406

407

408

409

410

411

412

413

414

415

416

417

418

419

420

421

422

423

424

425

426

427

428

429

430

431

432

433

434

435

436

437

438

439

440

441

442

Gradient ascent on $D$.

$$
\min _{\theta_{G}} \mathbb{E}_{I^{P} \sim P\left(I^{P}\right)} \log \left(1-D_{\theta_{D}}\left(G_{\theta_{G}}\left(I^{P}\right)\right)\right)
$$

Gradient descent on $G$.

Minimax problem allows discriminate to maximize adversarial networks, so that we can perform gradient ascent on these networks; whereas generator tries to minimize adversarial networks, so that we can perform gradient descent on these networks. In practice, Equation (6) might not provide enough gradients for $G$ to learn well. During the early stages of learning, when $G$ is poor, $D$ can reject samples with a high degree of confidence, since they are clearly different from the training data. In this case, $\log \left(1-D_{\theta_{D}}\left(G_{\theta_{G}}\left(I^{P}\right)\right)\right)$ saturates. As an alternative to training $G$ to minimize $\log \left(1-D_{\theta_{D}}\left(G_{\theta_{G}}\left(I^{P}\right)\right)\right.$ ), we can train $G$ to maximize $\log D_{\theta_{D}}\left(I^{F}\right)$. As a result of this objective function, the discriminator and generator have much stronger gradients at the start of the learning process.

When a single discriminating network is trained, the refiner network will tend to overemphasize certain features in order to fool the current discriminator network, resulting in drift and producing artifacts. We can conclude that any local patch sampled from the refined image should have similar statistics to the real image patches. We can therefore define a discriminator network that classifies each local image patch separately rather than defining a global discriminator network. The division limits both the receptive field and the capacity of the discriminator network, as well as providing a large number of samples per image for learning the discriminator network. The discriminator in our implementation is a fully convolutional network that outputs a probabilistic $N \times N$ ("N=2" represented as a synthesized face image) map instead of one scalar value to distinguish between a ground truth frontal view (GT) and a synthesized frontal view $(S F)$. Our discriminator loss is defined through the discrepancy between the model distribution and the data distribution, using an adversarial loss $\left(L_{a d v}\right)$. By assigning each probability value to a particular region, the $D_{\theta_{D}}$ can now concentrate on a single semantic region rather than the whole face.

\section{Comprehensive Loss Functions}

In addition to the existing TP-GAN synthesis loss functions, which are a weighted sum of four individual loss functions ( $L_{p x}, " L_{s y m}, L_{i p}, L_{t v}$ and $\left.L_{\text {local }} "\right)$, a classification loss $L_{\text {classify }}$ is further added to our method in order to get better results. Each individual loss function is presented below, and the used symbols are defined here. Let $I$ be an output image.

Let $W$ and $H$ be the width and height of $I,(x, y)$ be a pixel coordinate of a $2 \mathrm{D}$ image, $I^{\text {pred }}$ be the predicted (i.e., synthesized) frontal-face image of $I, I^{g t}$ be the representative frontal-face image 
443 of the ground-truth category of $I . I_{\text {local }}^{g t}$ be the composition image of the four local facial patches

444 of $I^{g t}$, and $I_{\text {local }}^{g t}$ be the composition image of the four local facial patches of $I$.

445

446

447

448

Pixel-Wise Loss $\left(L_{p x}\right)$

447

$$
L_{p x}=\frac{1}{W \times H} \sum_{x=1}^{W} \sum_{y=1}^{H}\left|I^{g t}(x, y)-I^{p r e d}(x, y)\right|
$$

449

450

451

Although pixel wise loss may bring some over-smooth effects to the refined results, it is still an

452

453 essential part for both accelerated optimization and superior performance.

454

455

\section{Symmetry Loss $\left(L_{s y m}\right)$}

456

457

458

459

460

461

$$
L_{\text {sym }}=\frac{1}{W / 2 \times H} \sum_{x=1}^{W / 2} \sum_{y=1}^{H}\left|I^{\text {pred }}(x, y)-I^{\text {pred }}(W-(x-1), y)\right|
$$

$L_{\text {sym }}$ is used to calculate the symmetry of the synthesized face image because a face image is generally considered to be a symmetrical pattern.

\section{Identity Preserving Loss $\left(L_{i p}\right)$}

462

461

463

464

465

466

467

468

469

470

471

472

473

$$
L_{i p}=\sum_{i=1}^{2} \frac{1}{W_{l} \times H_{l}} \sum_{x=1}^{W_{l}} \sum_{y=1}^{H_{l}}\left|F_{l}^{g t}(x, y)-F_{l}^{p r e d}(x, y)\right|
$$

where $F_{l}^{g t}$ and $F_{l}^{\text {pred }}$ respectively denote the feature map of the last two layers of the light-CNN net (Xiang et al., 2015) of $I^{g t}$ and $I^{\text {pred }}, W_{l}$ and $H_{l}$ are the width and height of the feature map of the last $l-t h$ layer $(l=1,2)$ of the light-CNN net. It is expected that a good synthesized frontalface image will have similar characteristics to its corresponding real frontal-face image. We employ a fully connected layer of the pre-trained light-CNN net for the feature extraction of the pre-trained recognition network. The pre-trained model will leverage the loss to enforce identitypreserving frontal view synthesis.

\section{Adversarial Loss $\left(L_{a d v}\right)$}


474

$$
L_{a d v}=\frac{1}{N} \sum_{n=1}^{N}-\log D_{\theta_{D}}\left(G_{\theta_{G}}\left(I_{n}^{P}\right)\right)
$$

475

$476 L_{a d v}$ is used to make the real frontal-face image $I^{F}$ and a synthesized frontal face images $G_{\theta_{G}}\left(I_{n}^{P}\right)$

477 indistinguishable, so that the synthesized frontal face image achieves a visually pleasing effect.

478

479 Total Variation Loss $\left(\boldsymbol{L}_{t v}\right)$

480 Generally, the face images synthesized by two pathways generative adversarial networks have 481 unfavorable visual artifacts, which deteriorates the visualization and recognition performance.

482 Imposing $L_{t v}$ on the final synthesized face images can help to alleviate this issue. The $L_{t v}$ loss is 483 calculated as follows:

484

485

$$
L_{t v}=\sum_{x=1}^{W} \sum_{y=1}^{H}\left|I_{x, y}^{\text {pred }}-I_{x-1, y}^{\text {pred }}\right|+\left|I_{x, y}^{\text {pred }}-I_{x, y-1}^{\text {pred }}\right|
$$

486

487

488

489

$L_{t v}$ will generate a smooth synthesized face image.

Classification Loss $\left(L_{\text {classify }}\right)$

490

491

$$
L_{\text {classify }}=-\sum_{i} y_{i}^{\text {true }} \log _{2}\left(y_{i}^{\text {pred }}\right)
$$

492

493

where $i$ is the class index, $y^{\text {true }}$ denotes the tensor of the one-hot true target of $I$, and $y^{\text {pred }}$ is the

494

495 predicted probability tensor. $L_{\text {classify }}$ is a cross-entropy loss which is used to ensure the

496

497 synthesized frontal-face image can be classified correctly.

\section{Local Pixel-Wise Loss $\left(L_{\text {local }}\right)$}

498

499

$$
L_{\text {local }}=\frac{1}{W \times H} \sum_{x=1}^{W} \sum_{y=1}^{H}\left|I_{\text {local }}^{g t}(x, y)-I_{\text {local }}^{\text {pred }}(x, y)\right|
$$

500

501 This loss is to calculate the total average pixel difference between $I_{\text {local }}^{g t}$ and $I_{\text {local }}^{\text {pred }}$, and it is not 502 addressed in (Rui et al., 2017) work. 
504 Generator Loss Functions ( $L_{\text {generator }}$ )

505 The generator loss function of the proposed method is a weighted sum of all the losses defined

506 above:

507

508

$$
L_{\text {generator }}=L_{p x}+\lambda_{1} L_{\text {sym }}+\lambda_{2} L_{i p}+\lambda_{3} L_{a d v}+\lambda_{4} L_{t v}+\lambda_{5} L_{\text {classyify }}+\lambda_{6} L_{\text {local }}
$$

509

510

where $\lambda_{i}(i=1 \sim 6)$ are weights that coordinate the different losses, and they are set to be

511

$\lambda_{1}=0.1, \lambda_{2}=0.001, \lambda_{3}=0.005, \lambda_{4}=0.0001, \lambda_{5}=0.1$, and $\lambda_{6}=0.3$ in our experiments. The

512

$L_{\text {generator }}$ is used to guide an identity-preserving inference of frontal view synthesis. While the

$513 L_{a d v}$ is used to push the generative network forward so the synthesized frontal face image

514 achieves a pleasing appearance.

515

\section{Optimizing the Training Process}

517 In order to optimize the training process, we propose some modifications to the TP-GAN

518 parameters as shown in Table 2 in order to improve the performance in learning the frontal-face

519 data distribution. In our experiment, we consider few parameters: learning rate, batch size,

520 number of epochs, and loss functions. We chose those parameters based on our experience,

521 knowledge and observations. The adopted learning rate improve the module loss accuracy for

522 both $G$ and $D$. The batch size is the number of examples from the training dataset used in the

523 estimation of the error gradient. This parameter determines how the learning algorithm will

524 behave. We have found that using a larger batch size has adversely affected our method

525 performance. As a result, during initial training the discriminator may be overwhelmed by too

526 many examples. This will lead to poor training performance. The number of training epochs is a

527 key advantage of machine learning. As the number of epochs increases, the performance will be

528 improved and the outcomes will be astounding. However, the disadvantage is that it takes a long

529 time to train a large number of epochs. These parameters are essential to improve LFMTP-

530 GAN's representation learning, gaining high-precision performance and reducing visual artifacts

531 when synthesising frontal-face images.

532

533 Experiments

534 We conducted extensive experiments to verify the effectiveness of our method by comparing it

535 with the TP-GAN. The evaluation protocol includes frontal face image resolution and accuracy

536 preserving face identity.

537

\section{Experimental Settings}


539 Both LFMTP-GAN and TP-GAN models are tested and trained on the Multi-PIE and FEI 540 datasets. Multi-PIE (Ralph et al., 2010) is a large face dataset with $75000+$ images for 337

541 identities across a variety of different poses, illuminations and expression conditions captured in

542 a constrained environment. Multi-PIE has 15 poses ranging from $\pm 90^{\circ}$, and 20 illumination

543 levels for each subject. All 20 illuminations were taken within a few seconds: two without any

544 flash illumination, followed by an 18 image with each flash firing independently. Figure 5 shows

545 an example of our model results. "The intensity of light is determined by the brightness of the

546 flash and the background. For example, a bright or dark flash, shadow reflection, or a white or

547 blue background will affect intensity. This depends on the recording equipment and the

548 positioning." To minimize the number of saturated pixels in flash illuminated images, all

549 cameras have been set to have a pixel value of 128 for the brightest pixel in an image without

550 flash illumination. In the same way, the diffusers in front of each flash were added. The color

551 balance was also manually adjusted so that the images looked similar. FEI is a Brazilian

552

553 unlabeled face dataset with $2800+$ images for 200 identities across a variety of different poses captured in a constrained environment. The face images were taken between "June 2005 and

554

555

556

557

558

559

560

561

\section{Visual Quality}

563

564

565

566

567

568

569

570

571

572

573

574

575

576

577

March 2006" at the artificial intelligence laboratory at são bernardo do campo, são paulo, brazil. The FEI images were taken against a white homogenous background in an upright frontal position with a $\pm 90^{\circ}$ range of profile poses; and different illuminations, distinct appearances and hairstyles were included for each subject. Our method shares the same implementation concept as TP-GAN but totally has different parameters settings. The training lasts for 10-to-18 days in each system for each dataset. The training model and source code will be released after this article is accepted.

In this subsection, we compare LFMTP-GAN with TP-GAN. Figure 6 and Fig 7 shows the comparison images, where the first column is the profile-face images under different face poses, the second column is the synthesized frontal-face images by TP-GAN, the third column is the synthesized frontal-face images by LFMTP-GAN, and the last column is one randomly selected frontal-face image of the category corresponding to the profile-face image. The yaw angle of the input face image are chosen circularly from $\left(15^{0}, 30^{0}, 45^{0}, 60^{0}, 75^{0}\right.$ and $\left.90^{0}\right)$. Obviously, the resolution of the LFMTP-GAN images looks better than TP-GAN. This reveals that the use of a proper 2D landmark localization algorithm could significantly improve the image quality of the synthesized images, provide rich textural details, and contain fewer blur effects. Therefore, the visualization results shown in Fig 6 and Fig 7 demonstrate the effectiveness of our method across a variety of poses and datasets. Similarly, Fig 8 shows the results of these datasets in close-up image with $90^{\circ}$ facial pose.

Previous frontal view synthesis methods are usually based on a posture range of $\pm 60^{\circ}$. It is generally believed that if the posture is greater than $60^{\circ}$, it is difficult to reconstruct the image of the front view. Nonetheless, we will show that with enough training data and a properly designed

Peer) Comput. Sci. reviewing PDF | (CS-2021:10:66723:2:0:NEW 25 Jan 2022) 
578 loss function, this is achievable. In Fig 9 and Fig 10, we show that LFMTP-GAN can recover 579 identity-preserving frontal faces from any pose, as well as comparing with state-of-the-art face

580 frontalization methods, it performs better. In addition, our geometry estimation method does not 581 require 3D geometry knowledge because it is driven by data alone.

582

583

584

585

586

587

588

589

590

591

592

593

594

595

596

597

598

599

600

601

602

603

604

605

606

607

\section{Identity Preserving}

To quantitatively demonstrate the identity preserving ability of the proposed method, we evaluate the classification accuracy of synthesized frontal-face images on both Multi-PIE and FEI databases, and show their classification accuracy (\%) in Table 3 across views and illuminations. The experiments were conducted by first employing light-CNN to extract deep features and then using the cosine-distance metric to compute the similarity of these features. The light-CNN model was trained on MS-Celeb-1M (Microsoft Celeb, 2016) which is a largescale face dataset, and fine-tuned on the images from Multi-PIE and FEI. Therefore, the lightCNN results on the profile images $I^{P}$ serves as our baseline. Our method produces better results than TP-GAN as the pose angle is increased. Our approach has shown improvements in frontalfrontal face recognition. Moreover, Table 4 illustrates that our method is superior to many existing state-of-the-art approaches.

Many deep learning methods have been proposed for frontal view synthesis, but none of them have been proved to be sufficient for recognition tasks. Chao et al., 2015; Yibo et al., 2018 relied on direct methods such as $\mathrm{CNN}$ for face recognition, which will definitely reduce rather than improve performance. It is therefore important to verify whether our synthesis results can improve recognition performance (whether "recognition via generation" works) or not. The next section presents the loss curves for the two models.

\section{Model Loss Curve Performance}

This section provides a comparison with TP-GAN. We analyze the effects of our model on three tradeoff parameters named generator loss, pixel-wise loss, and identity preserving accuracy. $80 \%$ of the face image subjects from the Multi PIE and FEI datasets were used for training and evolution purposes. $90 \%$ of the image subjects were used for training, while $10 \%$ were used for

608 testing. The recognition accuracy and corresponding loss curves are shown in Fig 10. We can

609 clearly see from the curves that, the proposed method improves the TP-GAN model and provides

610 the loss performance decreased sharply in LFMTP-GAN model, while the loss performance 611 decreased slightly in the TP-GAN model. Our optimization learning curves was calculated 612 according to the metric by which the parameters of the model were optimized, i.e., loss. More 613 importantly, our method still produces visually convincing results (as shown in Fig 6, Fig 7 and 614 Fig 9) even under extreme face poses, its recognition performance is about $1.2 \%$ higher than that 615 of TP-GAN.

616 


\section{Results and Discussions}

618 The goal of this method is to match the appearance of each query face by marking the partially

619 face surface of the generated image, such as the eyes, nose and mouth. In theory, this would have 620 allowed the TP-GAN method to better preserve facial appearance in the updated, synthesized

621 views. The statement holds true when the face is considered to have similar shape characteristics.

622 Considering that all human faces have unique shape characteristics, this may actually be

623 counterproductive and harmful, rather than improving face recognition. We believe that it is

624 necessary to integrate the four important local areas (eyes, nose and mouth) into their right

625 positions on the whole face image, which is already proved to be correct from our experiment

626 results. A majority of face recognition systems require a complete image to be recognized,

627 however, recovering the entire image is difficult when parts of the face are missing. This makes

628 it difficult to achieve good performance. We demonstrate how our approach can help enhance

629 face recognition by focusing on these areas of the face and outperform other methods in the same

630 context. Landmark detection is widely used in a variety of applications including object

631 detection, texture classification, image retrieval and etc. TP-GAN already has landmark detection

632 implemented for detecting the four face patches in its early stages as shown in Fig 1. Such a

633 method might be valuable in obtaining further textual details that can help in recovering those

634 facial areas and face shapes that have different characteristics. In our proposal, we offer a

635 relatively simple yet effective method for restoring the texture details of a synthesised face image

636 by repositioning the appearance areas of four landmark patches rather than the entire face. We

637 show a different method for facial recognition (Table 4) for faces with extreme poses. In four

638 major poses we achieve rank-1 recognition rates $(75 \%, 60 \%, 45 \%$, and 30\%). Furthermore,

639 when it comes to global $\left\{G_{\theta_{E}^{g}}, G_{\theta_{D}}\right\}$, we found that optimizing for the corrupted images resulted

640 in a better convergence rate than optimizing for the clean images in order to achieve balanced

641 learning behavior. Therefore, we neither extensively modify the $\left\{G_{\theta_{E}^{g}}, G_{\theta_{D}}\right\}$, nor include an

642 external neural network in our modification, because that integration will increase the network

643 complexity and cause training limitations. We obtained better results by introducing noise to the

644 image before feeding it to the $\left\{G_{\theta_{E}{ }^{\prime}} G_{\theta_{D}}\right\}$ during optimization.

645

646 Conclusion

647 In this paper, we propose an LFM model for synthesizing a frontal-face image from a single 648 image to further enhance the frontal-face images quality of the TP-GAN model. To accomplish

649 our goal smoothly, we expand the existing generative global pathway with a well-constructed 2D

650 face landmark localization to cooperate with the local pathway structure in a landmark sharing

651 manner to incorporate empirical face pose into the learning process, and improve the encoder-

652 decoder global pathway structure for better facial image features representation. Compared with

653 TP-GAN, our method can generate frontal images with rich texture details and preserve the

654 identity information. Face landmark localization allows us to restore the missing information of 
655 the real face image from the synthetic frontal images, and provide a rich texture detail. The

656

657

658

659

660

661

662

663

664

665

666

667

668

669

670

671

672

673

674

675

676

677

678

679

680

681

682

683

684

685

686

687

688

689

690

691

692

quantitative and qualitative experimental results of the Multi-PIE and FEI datasets show that our proposed method can not only generate high-quality perceptual facial images in extreme poses, but also significantly improves the TP-GAN results. Although LFMTP-GAN method achieves a high-quality image resolution output, there is still room for improvement by choosing different optimization algorithms, such as loss functions, or introducing some different techniques for facial analysis and recognition. Our future research is to apply different error functions or different face analysis and recognition techniques, combined with two pathway structures, to achieve a super-resolution generative model and high-precision performance.

\section{References}

Junho Y, Heechul J, ByungIn Y, Changkyu C, Dusik P, Junmo K. 2015. Rotating Your Face Using Multi-Task Deep Neural Network. In: 2015 IEEE Conference on Computer Vision and Pattern Recognition (CVPR). Boston, MA, USA: 676 - 684 DOI:

10.1109/CVPR.2015.7298667.

Chao X, Xiaowei Z, Danhang T, Karlekar J, Shuicheng Y, Tae-Kyun K. 2015. Conditional

Convolutional Neural Network for Modality-aware Face Recognition. In: 2015 IEEE International Conference on Computer Vision (ICCV). Santiago, Chile: 3667 - 3675 DOI: 10.1109/ICCV.2015.418.

Xi Y, Xiang Y, Kihyuk S, Xiaoming L, Manmohan C. 2017. Towards Large-Pose Face Frontalization in the Wild. In: 2017 IEEE International Conference on Computer Vision (ICCV). Venice, Italy: 3990 - 3999 DOI: 10.1109/ICCV.2017.430.

Jian Z, Yu C, Yan X, Lin X, Jianshu L, Fang Z, Karlekar J, Sugiri P, Shengmei S, Junliang X, Shuicheng Y, Jiashi F. 2018. Towards Pose Invariant Face Recognition in the Wild. In: 2018 IEEE/CVF Conference on Computer Vision and Pattern Recognition. Salt Lake, UT, USA: 2207 - 2216 DOI: 10.1109/CVPR.2018.00235.

Bassel Z, Ilya K, Yuri M. 2021. PFA-GAN: Pose Face Augmentation Based on Generative Adversarial Network. Vilnius University, vol. 32, no. 2: 425 - 440 DOI: https://doi.org/10.15388/21-INFOR443

Chenxu Z, Saifeng N, Zhipeng F, Hongbo L, Ming Z, Madhukar B, Xiaohu G. 2021. 3D

Talking Face with Personalized Pose Dynamics. In: IEEE Transactions on Visualization and Computer Graphics. 1 - 25 DOI: 10.1109/TVCG.2021.3117484.

Yi Z, Keren F, Cong H, Peng C. 2021. Identity-and-pose-guided Generative Adversarial Network for Face Rotation. 33 - 47 DOI: 10.1016/J.NEUCOM.2021.04.007.

Hang Z, Jihao L, Ziwei L, Yu L, Xiaogang. 2020. Rotate-and-Render: Unsupervised Photorealistic Face Rotation from Single-View Images. In: 2020 IEEE/CVF Conference on Computer Vision and Pattern Recognition (CVPR). Seattle, WA, USA: 5911 - 5920 DOI: 10.1109/CVPR42600.2020.00595. 
693 Yibo H, Xiang W, Bing Y, Ran H, Zhenan S. 2018. Pose-Guided Photorealistic Face

694 Rotation. In: 2018 IEEE/CVF Conference on Computer Vision and Pattern Recognition. Salt

695 Lake City, UT, USA: 18 - 23 DOI: 10.1109/CVPR.2018.00876.

696 Luan T, Xi Y, Xiaoming L. 2018. Representation Learning by Rotating Your Faces. IEEE

697 Transactions on Pattern Analysis and Machine Intelligence, vol. 41, 1s. 12: 3007 - 3021 DOI:

698 10.1109/TPAMI.2018.2868350.

699 Rui H, Shu Z, Tianyu L, Ran H. 2017. Beyond Face Rotation: Global and Local Perception

700 GAN for Photorealistic and Identity Preserving Frontal View Synthesis. In: 2017 IEEE

701 International Conference on Computer Vision (ICCV). Venice, Italy: 2439 - 2448 DOI:

$702 \quad 10.1109 /$ ICCV.2017.267.

703 Soumyadip S, Jun-Cheng C, Carlos C, Vishal M. P, Rama C, David W J. 2016. Frontal to

704 Profile Face Verification in The Wild. In: 2016 IEEE Winter Conference on Applications of

705 Computer Vision (WACV). Lake Placid, NY, USA: 1 - 9 DOI:

$706 \quad$ 10.1109/WACV.2016.7477558.

707 Peipei L, Xiang W, Yibo H, Ran H, Zhenan S. 2019. M2fpa: A multi-yaw Multi-Pitch High-

708 Quality Database and Benchmark for Facial Pose Analysis: 1 - 9 Available at

709 https://arxiv.org/abs/1904.00168

710 Meina K, Shiguang S, Hong C, Xilin C. 2014. Stacked Progressive Auto-Encoders (SPAE)

711 for Face Recognition Across Poses. In: 2014 IEEE Conference on Computer Vision and

712 Pattern Recognition. Columbus, OH, USA: 1883 - 1890 DOI: 10.1109/CVPR.2014.243.

713 Tal H, Shai H, Eran P, Roee E. 2015. Effective Face Frontalization in Unconstrained Images.

714 In: 2015 IEEE Conference on Computer Vision and Pattern Recognition (CVPR). Boston,

715 USA: 1 - 10 Available at https://arxiv.org/abs/1411.7964

716 Christos S, Yannis P, Stefanos Z, Maja P. 2015. Robust Statistical Face Frontalization. In:

7172015 IEEE International Conference on Computer Vision (ICCV). Santiago, Chile: 3871 -

7183879 DOI: $10.1109 / \mathrm{ICCV} .2015 .441$.

719 Omkar M P, Andrea V, Andrew Z. 2015. Deep Face Recognition. In: Proceeding of the

720 British Machine Vision Conference (BMVC). England, UK: 1 - 12 DOI: 10.5244/C.29.41.

721 BMVA Press.

722 Florian S, Dmitry K, James P. 2015. FaceNet: A Unified Embedding for Face Recognition

723 and Clustering. In: 2015 IEEE Conference on Computer Vision and Pattern Recognition

724 (CVPR). Boston, MA, USA: 1 - 10 DOI: 10.1109/CVPR.2015.7298682.

725 John G D. 1985. Uncertainty Relation for Resolution in Space, Spatial Frequency, and

726 Orientation Optimized by Two-dimensional Visual Cortical Filters. Journal of Optical

727 American, vol. 2, 1s. 7: 1160 - 1169 DOI: 10.1364/josaa.2.001160.

728 David - G Lowe. 1999. Object Recognition from Local Scale-invariant Features. In:

729 Proceeding of the $7^{\text {th }}$ IEEE International Conference on Computer Vision. Kerkyra, Greece:

$7301150-1157$ DOI: 10.1109/ICCV.1999.790410. 
731

732

733

734

735

736

737

738

739

740

741

742

743

744

745

746

747

748

749

750

751

752

753

754

755

756

757

758

759

760

761

762

763

764

765

766

767

768

769

Ahonen T, Hadid A, Pietikäinen M. 2006. Face Description with Local Binary Patterns: Application to Face Recognition. IEEE Transaction on Pattern Analysis and Machine Intelligence, vol. 28, no. 12: 2037 - 2041 DOI: 10.1109/TPAMI.2006.244.

Dalal N, Triggs B. 2005. Histograms of Oriented Gradients for Human Detection. In: 2005 IEEE Computer Society Conference on Computer Vision and Pattern Recognition (CVPR'05). San Diego, CA, USA: 886 - 893 DOI: 10.1109/CVPR.2005.177.

Kilian Q W, Lawrence K S. 2009. Distance Metric Learning for Large Margin Nearest Neighbor Classification. Journal of Machine Learning Research (2009) 207-244, vol. 10: 1 38.

Tsai-Wen C, Trevor J W, Yi S, Stefan R P, Sabine L R, Amy B, Eric R S, Rex A K, Michael B O, Vivek J, Loren L Karel S, Douglas S K. 2013. Ultrasensitive Fluorescent Proteins for Imaging Neuronal Activity. Journal Natural Research, vol. 499, Is. 7458: 295 - 300 DOI: 10.1038/nature12354.

Yaniv T, Ming Y, Marc'Aurelio R, Lior W. 2014. DeepFace: Closing the Gap to HumanLevel Performance in Face Verification. In: 2014 IEEE Conference on Computer Vision and Pattern Recognition (CVPR). Columbus, OH, USA: 1 - 8 DOI: 10.1109/CVPR.2014.220.

Amin J, Xiaoming L. 2017. Pose-invariant Face Alignment via CNN-based Dense 3D Model Fitting. International Journal of Computer Vision, vol. 125, no. 2: 187 - 203 DOI:

10.1109/CVPR.2016.454.

Kaipeng Z, Zhanpeng Z, Zhifeng L, Yu Q. 2016. Joint Face Detection and Alignment using Multi-Task Cascaded Convolutional Networks. In: Proceeding of the $4^{\text {th }}$ International Conference on Information Science and Control Engineering (ICISCE). Changsha, China: 15 DOI: 10.1109/ICISCE.2017.95

Jimei Y, Scott R, Ming-Hsuan Y, Honglak L. 2016. Weakly-supervised Disentangling with Recurrent Transformations for 3D View Synthesis. In: Proceeding of the $28^{\text {th }}$ International Conference on Neural Information Processing Systems, vol. 1: 1099 - 1107. Luan T, Xi Y, Xiaoming L. 2017. Disentangled Representation Learning GAN for Pose invariant Face Recognition. In: 2017 IEEE Conference on Computer Vision and Pattern Recognition (CVPR). Honolulu, HI, USA: 1415 - 1424 DOI: 10.1109/CVPR.2017.141.

Xiang W, Ran H, Zhenan S, Tieniu T. 2015. A light CNN for Deep Face Representation with Noisy Labels. IEEE Transactions on Information Forensics and Security, vol. 13, 1s. 11: 2884 - 2896 DOI: 10.1109/TIFS.2018.2833032.

Yu T, Xi P, Long Z, Shaoting Z, Dimitris N M. 2018. CR-GAN: Learning Complete Representations for Multi-View Generation. In: Proceeding of the $27^{\text {th }}$ International Joint Conference on Artificial Intelligence (IJCAI-18): 942 - 948 Available at https://arxiv.org/abs/1806.11191v1

Yu Y, Songyao J, Joseph P R, Yun F. 2020. Dual-Attention GAN for Large-pose Face Frontalization. In: $202015^{\text {th }}$ IEEE International Conference on Automatic Face and Gesture Recognition (FG2020): 24 - 31 DOI: 10.1109/FG47880.2020.00004.

Peer] Comput. Sci. reviewing PDF | (CS-2021:10:66723:2:0:NEW 25 Jan 2022) 
770

771

772

773

774

775

776

777

778

779

780

781

782

783

784

785

786

787

788

789

790

791

792

793

794

795

796

797

798

799

800

801

802

803

804

805

806

807

808

Ian J G, Jean P A, Mehdi M, Bing X, David W F, Sherjil O, Aaron C, Yoshua B. 2014. Generative Adversarial Networks. In: International Conference on Neural Information Processing Systems (NIPS): 2672 - 2680 Available at https://arxiv.org/abs/1406.2661 Christian L, Lucas T, Ferenc H, Jose C, Andrew C, Alejandro A, Andrew A, Alykhan T, Johannes T, Zehan W, Wenzhe S. 2017. Photo-Realistic Single Image Super-Resolution Using a Generative Adversarial Network. In: 2017 IEEE Conference on Computer Vision and Pattern Recognition (CVPR). Honolulu, HI, USA: 1 - 19 DOI: 10.1109/CVPR.2017.19. Alec R, Luke M, Soumith C. 2016. Unsupervised Representation Learning with Deep Convolutional Generative Adversarial Networks: 1 - 16 Available at https://arxiv.org/pdf/1511.06434.pdf

Mehdi M, Simon O. 2014. Conditional Generative Adversarial Nets: 1411 - 1784 Available at https://arxiv.org/abs/1411.1784

Augustus O, Christopher O, Jonathon S. 2017. Conditional Image Synthesis with Auxiliary Classifier GANs. In: Proceeding of the $34^{\text {th }}$ International Conference on Machine Learning, (PMLR 70:2642-2651), vol. 70. Sydney, Australia: 2642 - 2651 Available at https://arxiv.org/abs/1610.09585

David B, Thomas S, Luke M. 2017. BEGAN: Boundary Equilibrium Generative Adversarial Networks: 1 - 10 Available at https://arxiv.org/abs/1703.10717

Anthreas A, Amos S, Harrison E. 2018. Data Augmentation Generative Adversarial

Networks: 1 - 14 Available at https://arxiv.org/abs/1711.04340

Pascal V, Hugo L, Yoshua B, Pierre-Antoine M. 2008. Extracting and Composing Robust Features with Denoising Autoencoders. In: Proceeding of the $25^{\text {th }}$ International Conference on Machine Learning, Helsinki, Finland: 1 - 8 DOI: 10.1145/1390156.1390294.

Jun-Yan Z, Taesung P, Phillip I, Alexei A E. 2017. Unpaired Image-to-Image Translation Using Cycle - Consistent Adversarial Networks. In: 2017 IEEE International Conference on Computer Vision (ICCV). Venice, Italy: 1 - 18 DOI: 10.1109/ICCV.2017.244.

Xiangyu Z, Zhen L, Junjie Y, Dong Y, Stan Z L. 2015. High-fidelity Pose and Expression Normalization for Face Recognition in The Wild. In: 2015 IEEE Conference on Computer Vision and Pattern Recognition (CVPR). Boston, MA, USA: 787 - 796 DOI:

10.1109/CVPR.2015.7298679.

Li L, Paul F, Guoying Z, Matti P. 2014. Extended Local Binary Pattern Fusion for Face Recognition. In: 2014 IEEE International Conference on Image Processing (ICIP). Paris, France: 718 - 722 DOI: 10.1109/ICIP.2014.7025144.

Xuehan X, Fernando De la T. 2013. Supervised Descent Method and Its Applications to Face Alignment. In: 2013 IEEE Conference on Computer Vision and Pattern Recognition (CVPR). Portland, OR, USA: 1 - 8 DOI: 10.1109/CVPR.2013.75.

Georgios T. 2015. Project-out Cascaded Regression with an Application to Face Alignment. In: 2015 IEEE Conference on Computer Vision and Pattern Recognition (CVPR). Boston, MA, USA: 3659 - 3667 DOI: 10.1109/CVPR.2015.7298989. 
809 Sanjeev A, Andrej R, Yi Z. 2018. Do GANs Learn the Distribution? Some Theory and 810 Empirics. In: Proceeding of $6^{\text {th }}$ International Conference on Learning Representations (ICLR 811 2018): 1 - 11 Available at https:/openreview.net/forum?id=BJehNfW0-

812 Cong H, Zhen-Hua F, Xiao-Jun W, Josef K. 2020. Dual Encoder-Decoder based Generative 813 Adversarial Networks for Disentangled Facial Representation Learning. IEEE Transactions, 814 vol. 8: 130159 - 130171 DOI: 10.1109/ACCESS.2020.3009512

815 Kevin R, Aurelien L, Sebastian N, Thomas H. 2017. Stabilizing Training of Generative 816 Adversarial Networks Through Regularization. In: Proceeding of the $31^{\text {st }}$ Conference on 817 Neural Information Processing Systems (NIPS 2017). Long Beach, CA, USA: 1 - 11 818 Available at https://arxiv.org/abs/1705.09367

819 Adrian B, Georgios T. 2017. How Far are we from Solving The 2D \& 3D Face Alignment 820 Problem? (and a Dataset of 230,000 3D Facial Landmarks). In: 2017 IEEE International 821 Conference on Computer Vision (ICCV). Venice, Italy: 1021 - 1030 DOI:

$822 \quad$ 10.1109/ICCV.2017.116.

823 Xing D, Vishwanath A. Sindagi, Vishal M-P. 2018. GP-GAN: Gender Preserving GAN for 824 Synthesizing Faces from Landmarks: 1 - 7 Available at https://arxiv.org/pdf/1710.00962.pdf 825 Justin J, Alexandre A, Li F-F. 2016. Perceptual Losses for Real-time Style Transfer and 826 Super-Resolution. In: Proceeding of $14^{\text {th }}$ European Conference on Computer Vision.

827 Amsterdam, Netherlands: 1 - 17 Available at https://arxiv.org/abs/1603.08155

828 Ralph G M, Jeffrey C, Takeo K, Simon B. 2010. Multi-PIE. Image and Vision Computing. In:

829 Proceeding International Conference Auto Face Gesture Recognition, vol. 28, no. 5: $1-23$

830 DOI: $10.1016 /$ j.imavis.2009.08.002.

831 FEI. 2005-2006. Artificial Intelligence Laboratory of FEI, São Bernardo do Campo, São

832 Paulo, Brazil: Available at https://fei.edu.br/ cet/facedatabase.html

833 Microsoft Celeb (MS-Celeb-1M). 2016: Available at https://www.microsoft.com/en-

834 us/research/project/ms-celeb-1m-challenge-recognizing-one-million-celebrities-real-world 
Figure 1

The structure of TP-GAN.

The final output was obtained by integrating the global pathway with a 2D facial landmark localization to collaborate with the local pathway in a landmark sharing fashion. The dataset was downloaded from the official TP-GNA GitHub page: https://github.com/HRLTY/TP- GAN.

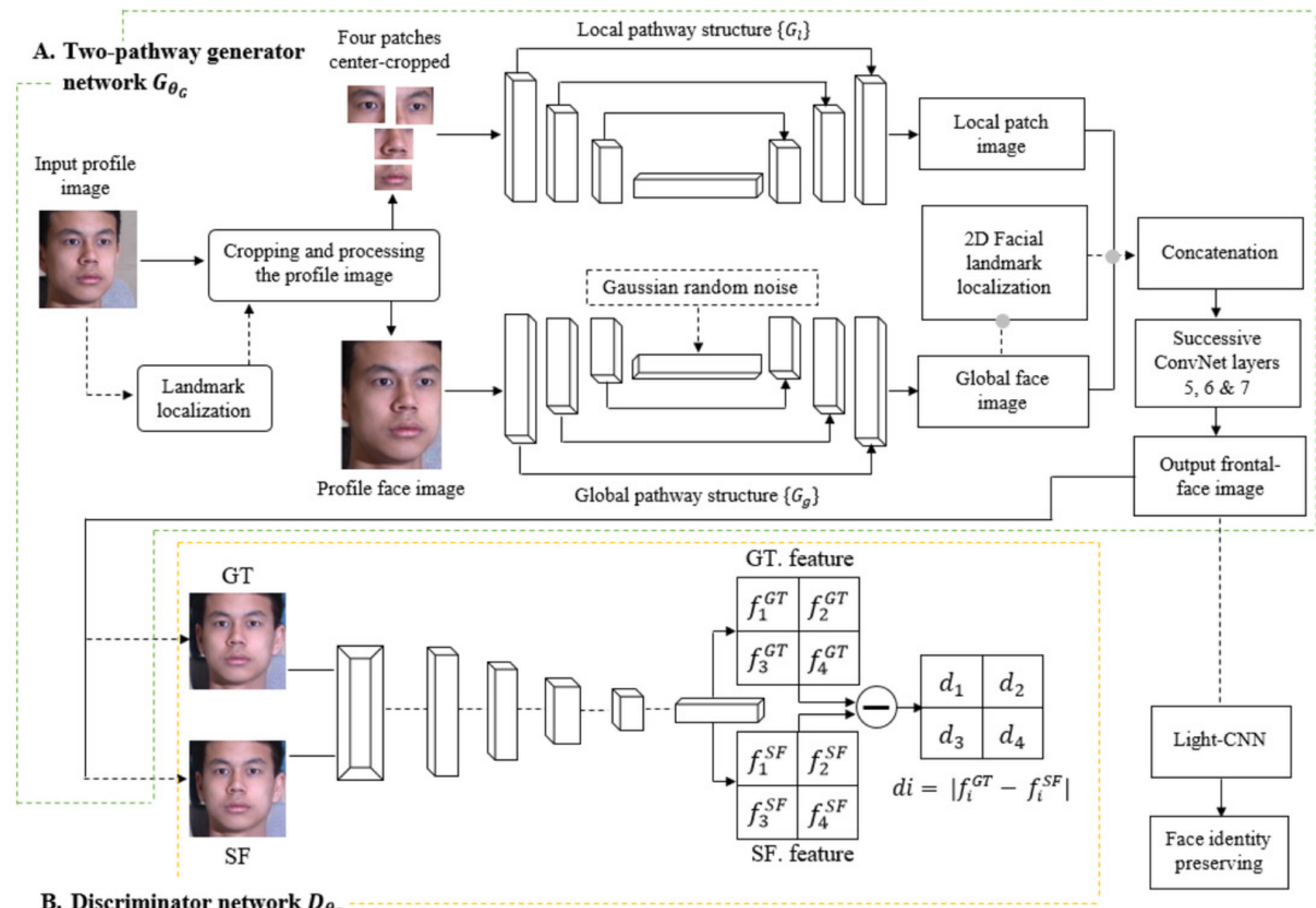


Figure 2

The structure of the 2D facial landmark localization method.

The dataset was downloaded from the official TP-GNA GitHub page:

https://github.com/HRLTY/TP- GAN.

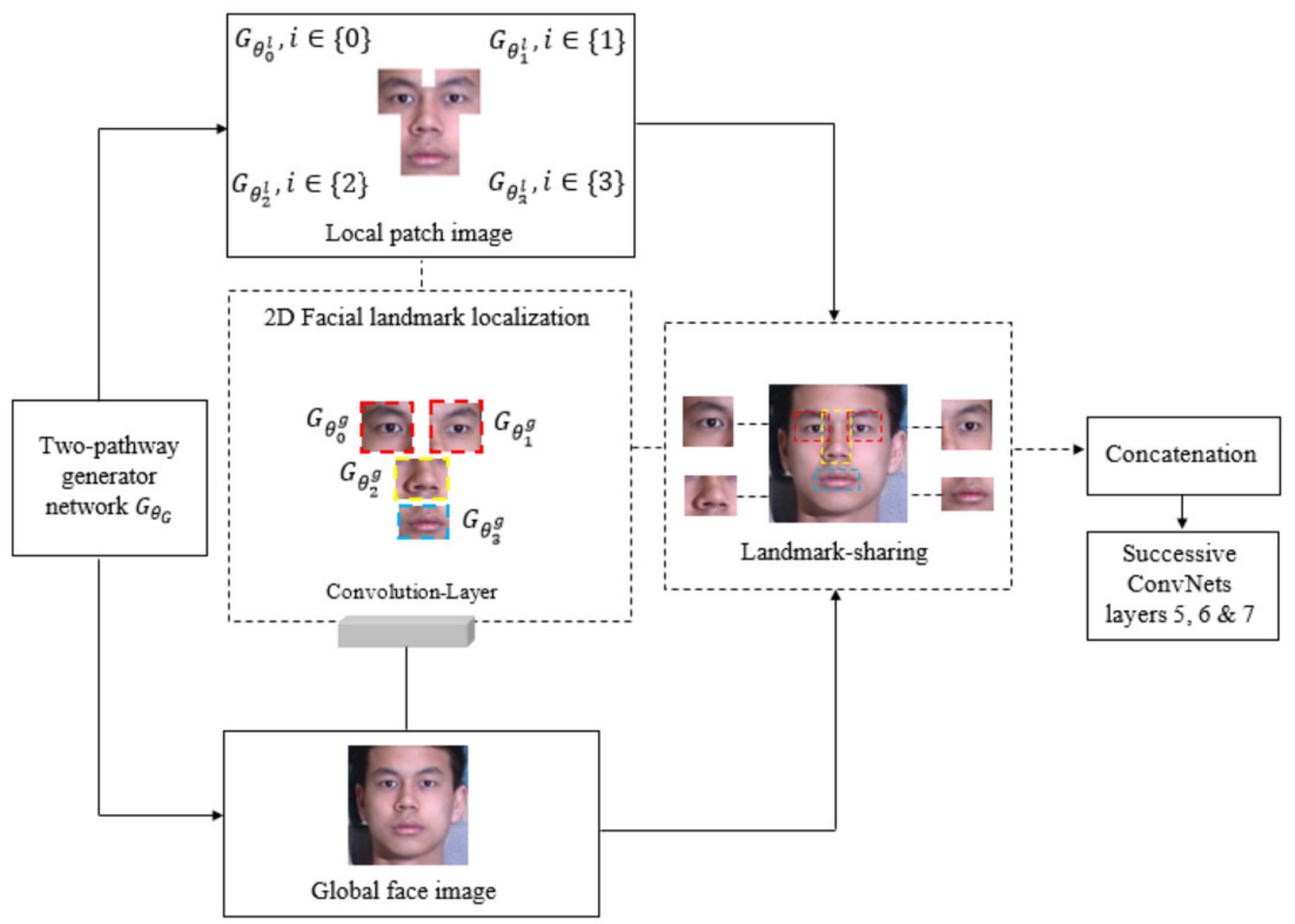


Figure 3

The detection of different facial shapes characteristics.

Example1., The appearance of the face is very accurate because all of the generated local synthesis patches (or templates) are centralized. In examples 2, 3, and 4 the appearance of the face is inaccurate since all of the generated local synthesis patches (or templates) are decentralized, resulting in mismatches or drifts. Faces in examples 2, 3, and 4 can be treated using our method regardless of their shape.

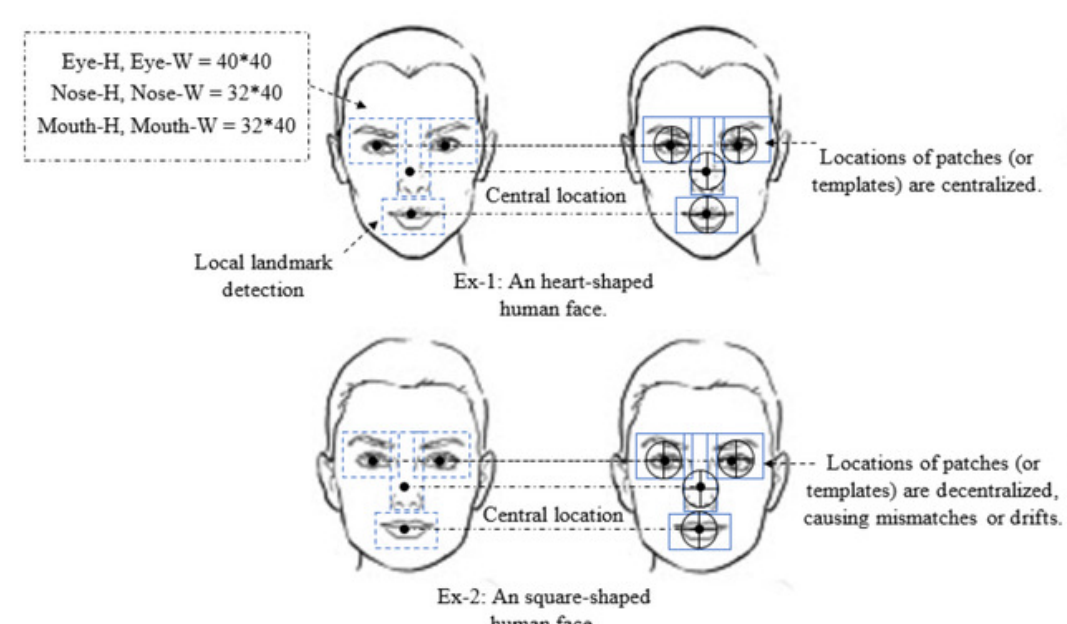

human face.

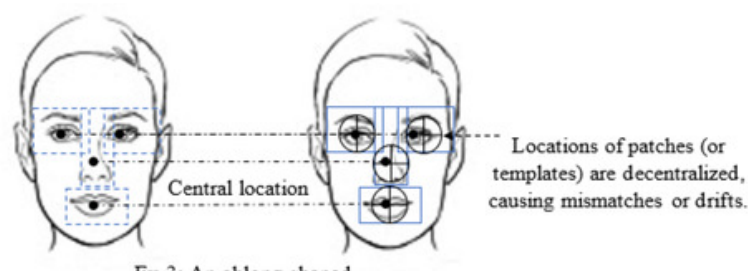

Ex-3: An oblong-shaped

human face.

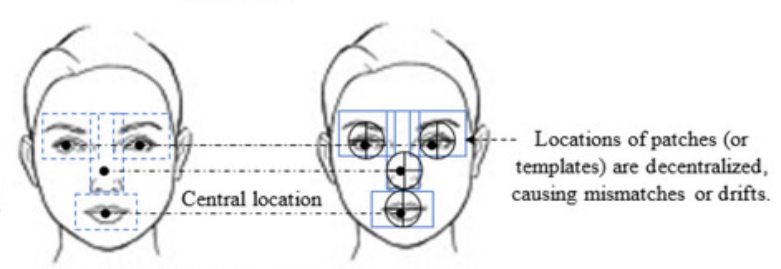

Ex-4: An round-shaped human face. 
Figure 4

The global pathway structure. The structure is based on encoder down-sampling and decoder up-sampling. The bottleneck with 256-D features vector remains the same.

The dataset was downloaded from the official FEI website: https://fei.edu.br/ cet/ facedatabase.html .

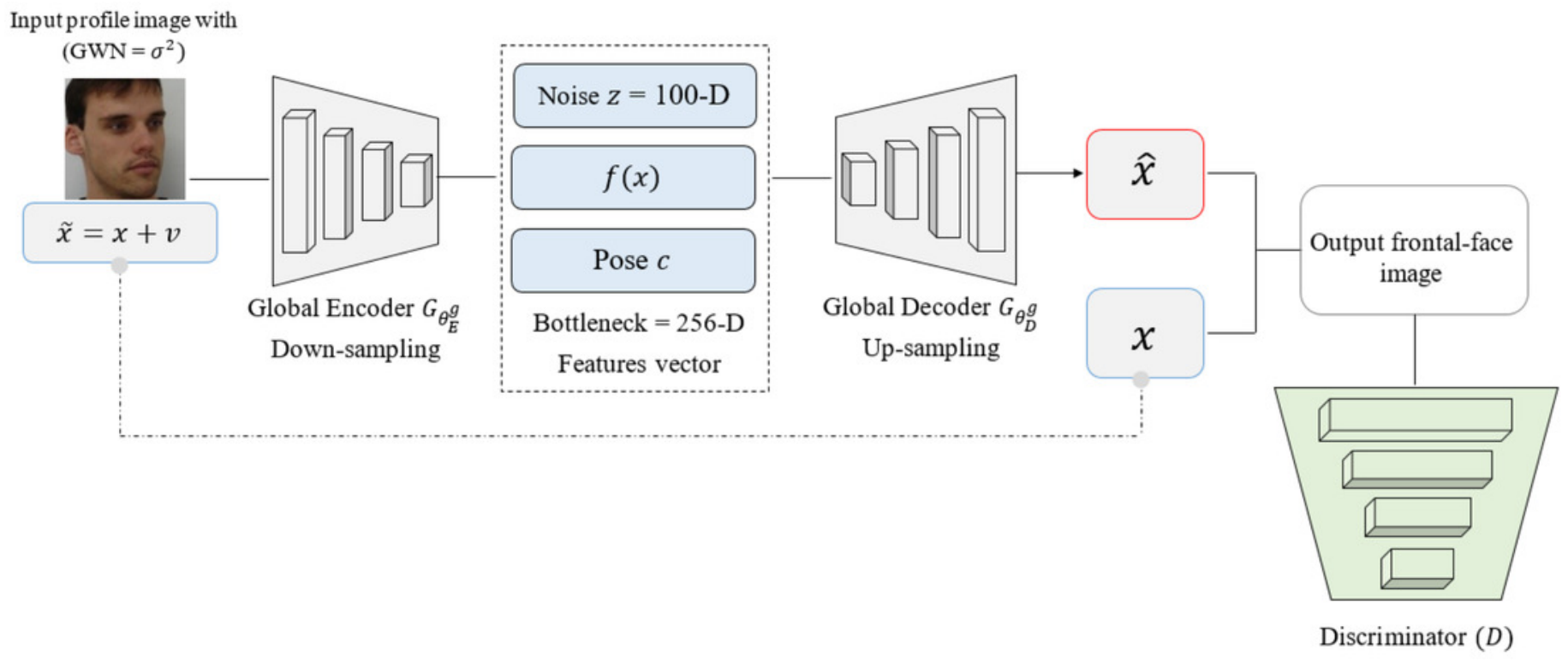




\section{Figure 5}

Examples of different levels of illumination.

The level of illumination, including brightness, exposure, contrast, and shadows and etc.

Some quality effects can also be observed, such as sharpness, smoothness, blurriness, etc.

The dataset was downloaded from the official TP-GNA GitHub page:

https://github.com/HRLTY/TP- GAN . 


\begin{tabular}{llccc}
\hline Pose range & $\begin{array}{l}\text { Profile } \\
\text { image }\end{array}$ & TP-GAN & LFMTP- & Ground- \\
& & GAN & Truth \\
\hline
\end{tabular}

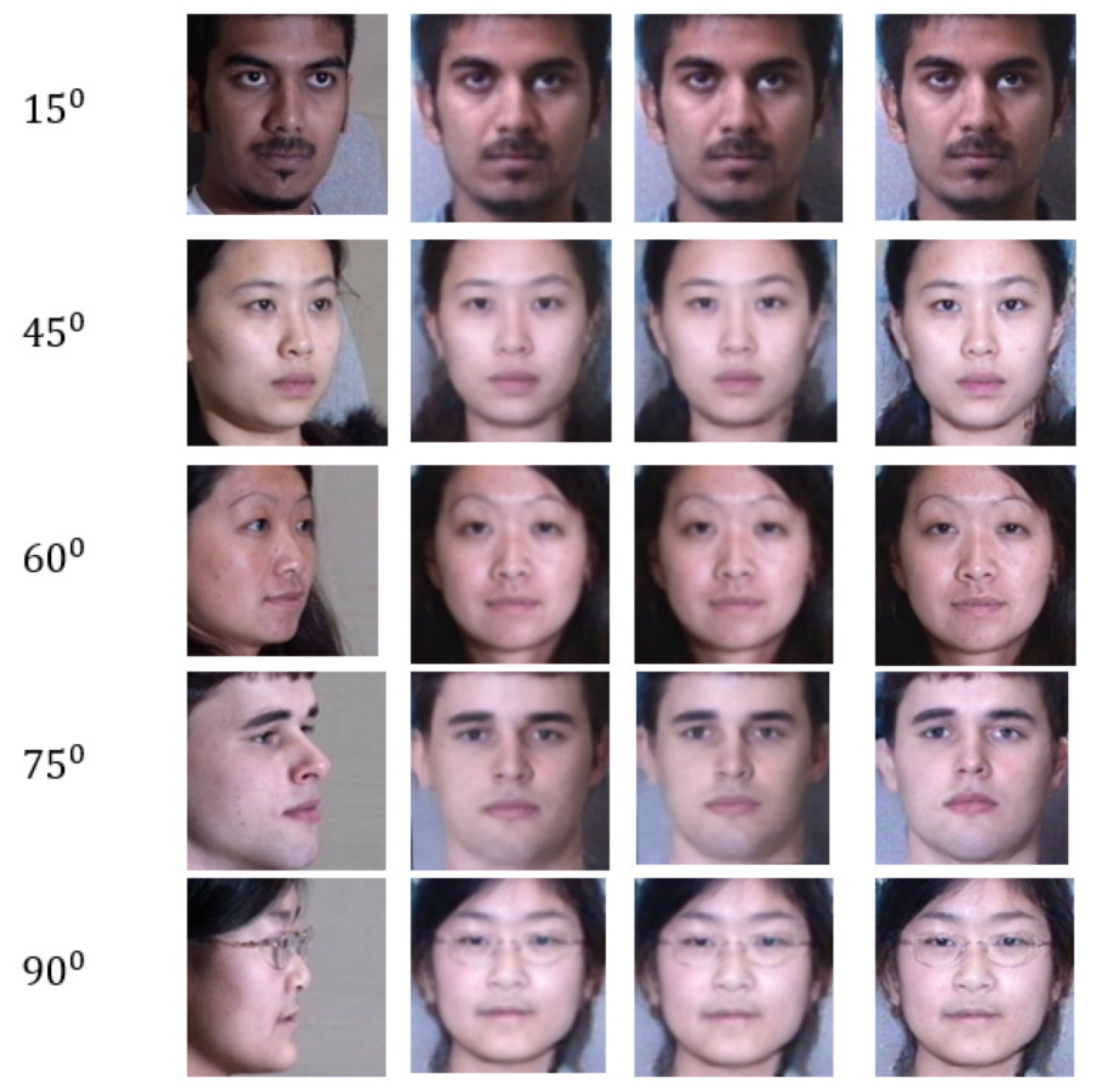




\section{Figure 6}

Comparison of TP-GAN and LFMTP-GAN by synthesizing frontal-face images under different poses and illuminations on Multi-PIE face database. It can be seen that LFMTPGAN generates better visual quality images.

The dataset was downloaded from the official TP-GNA GitHub page:

https://github.com/HRLTY/TP- GAN . 


Pose range Profile image TP-GAN LFMTP-GAN Ground-Truth

$15^{0}$

$30^{\circ}$
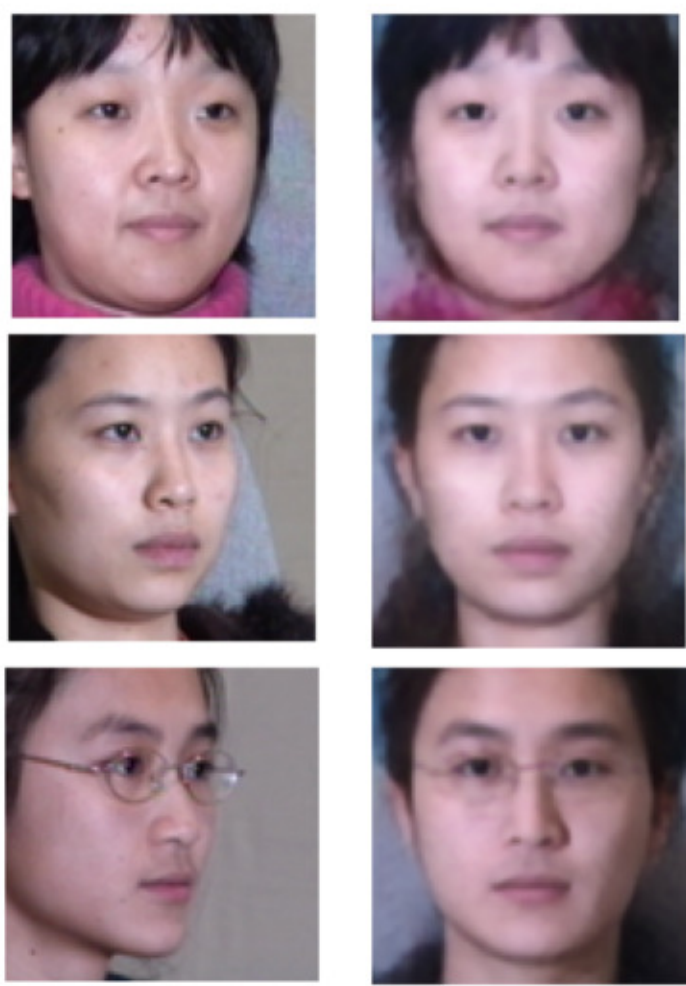

$45^{0}$

$60^{0}$
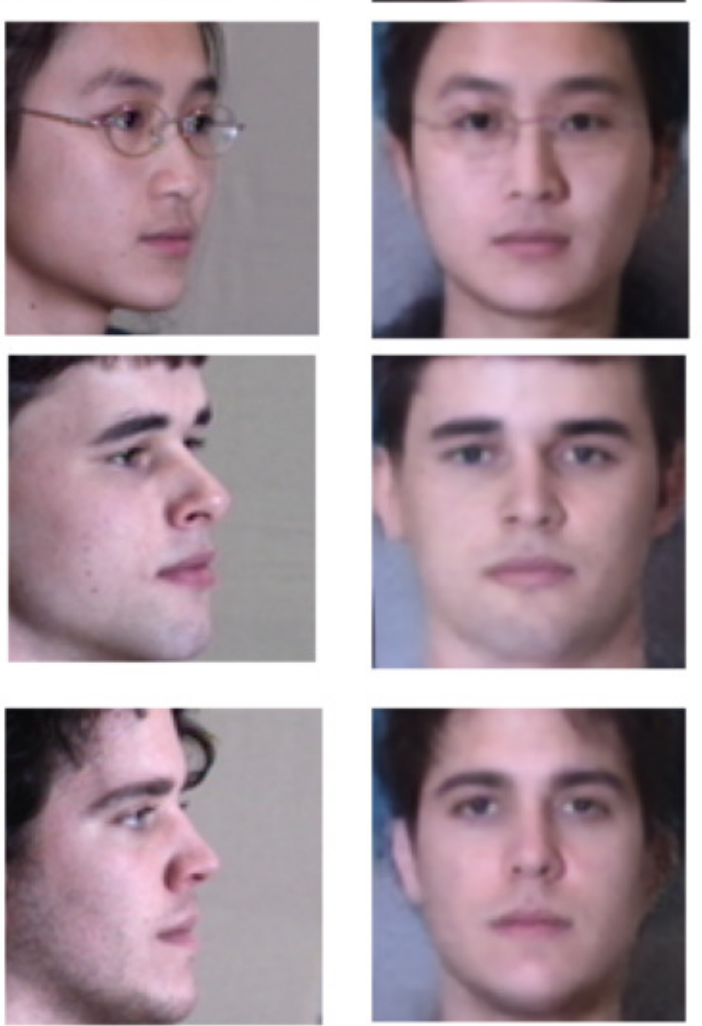

$75^{0}$
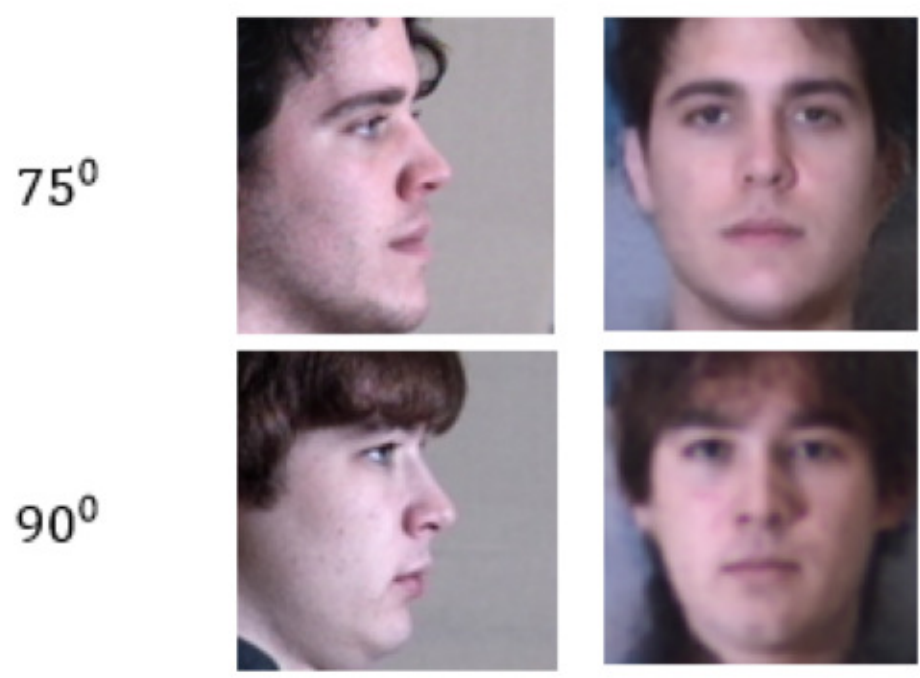
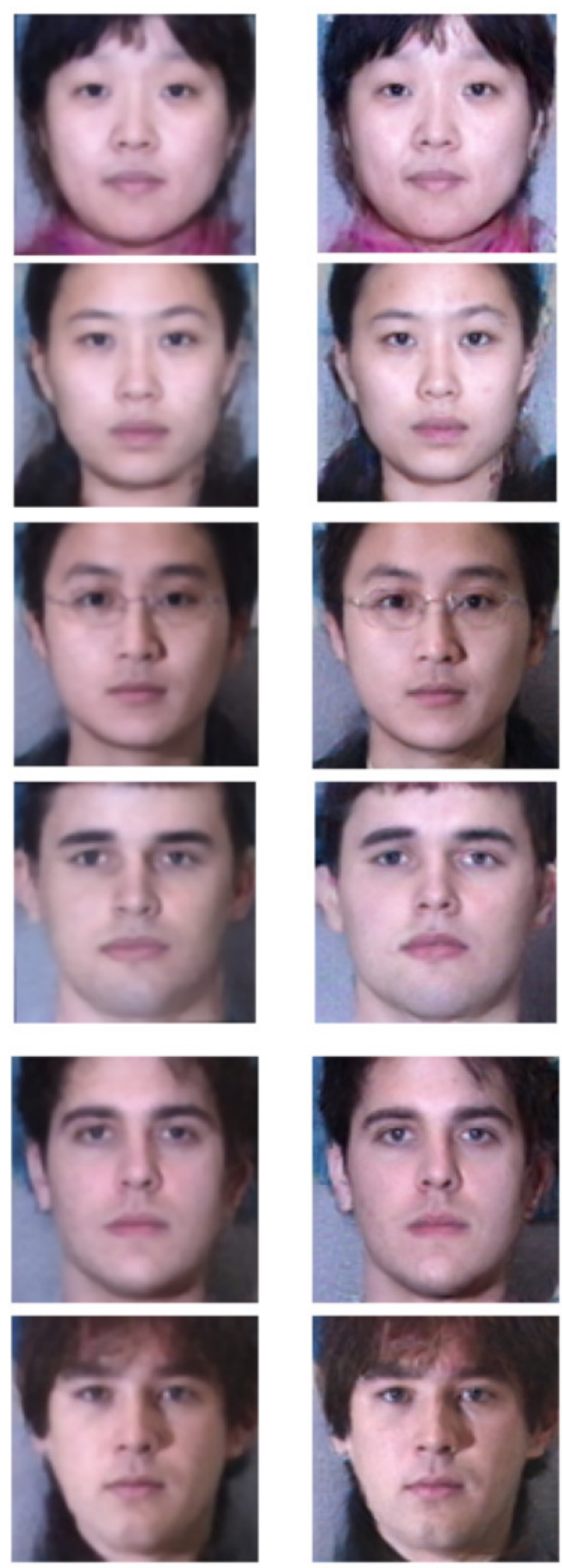


\section{Figure 7}

Comparison of TP-GAN and LFMTP-GAN by synthesizing frontal-face images under different poses and illuminations on FEl face database. It can be seen that LFMTP-GAN generates better visual quality images with less blurry visualization effect.

The dataset was downloaded from the official FEl website: https://fei.edu.br/ cet/ facedatabase.html . 


Pose range Profile image TP-GAN LFMTP-GAN Ground-Truth
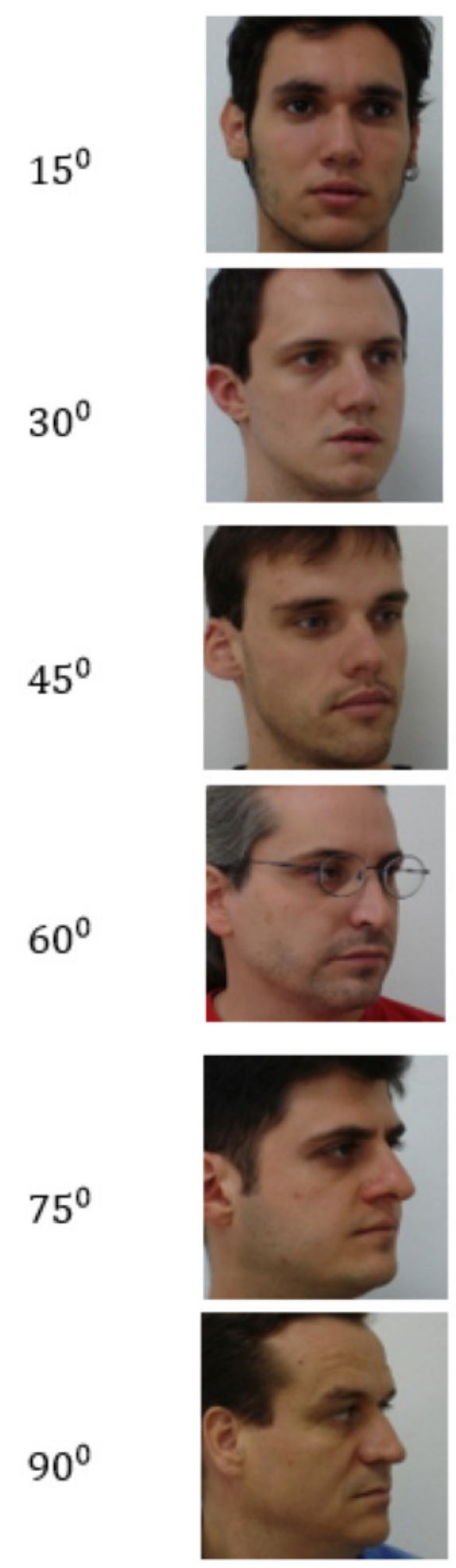
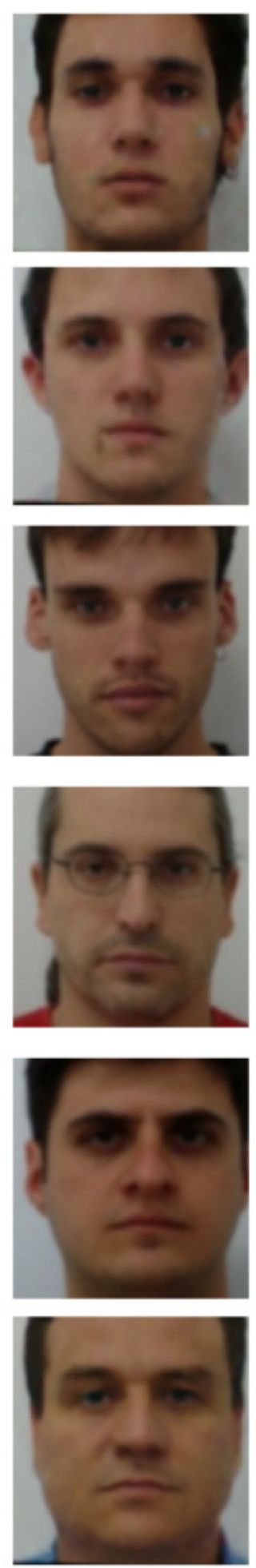
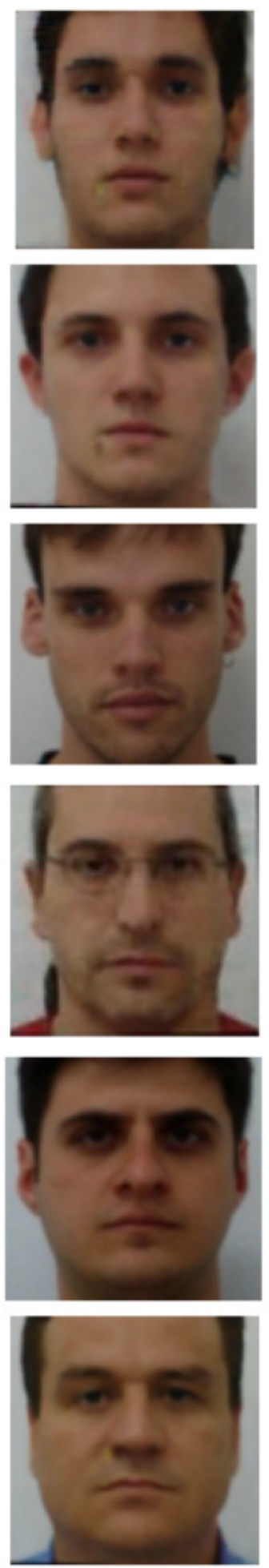
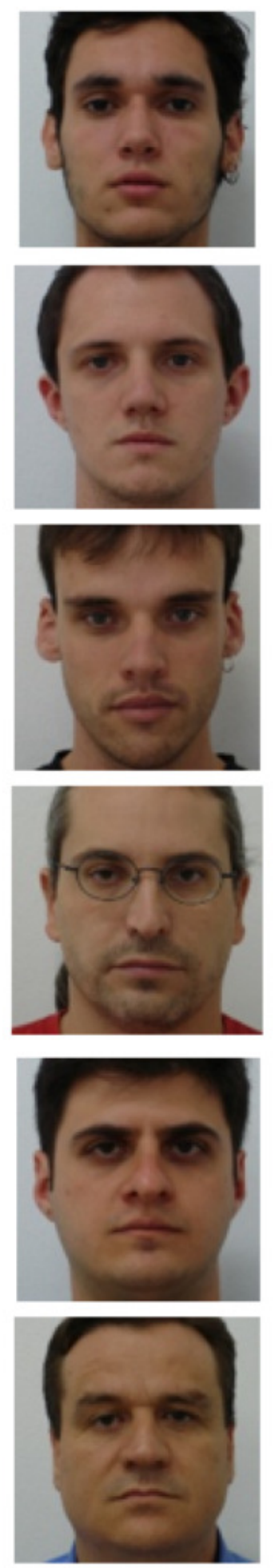


\section{Figure 8}

A comparison of the resolution of facial images taken under $90^{\circ}$ face pose, and illuminations condition. The facial visualization area is inside the yellow map.

The dataset was downloaded from the official websites: https://github.com/HRLTY/TP- GAN and https://fei.edu.br/ cet/ facedatabase.html .

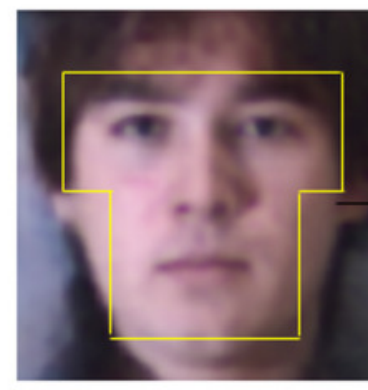

TP-GAN on MultiPIE Frontal-face $\left(90^{\circ}\right)$

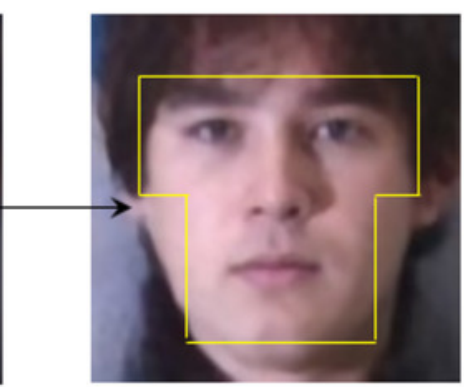

LFMTP-GAN on Multi-PIE Frontalface $\left(90^{\circ}\right)$

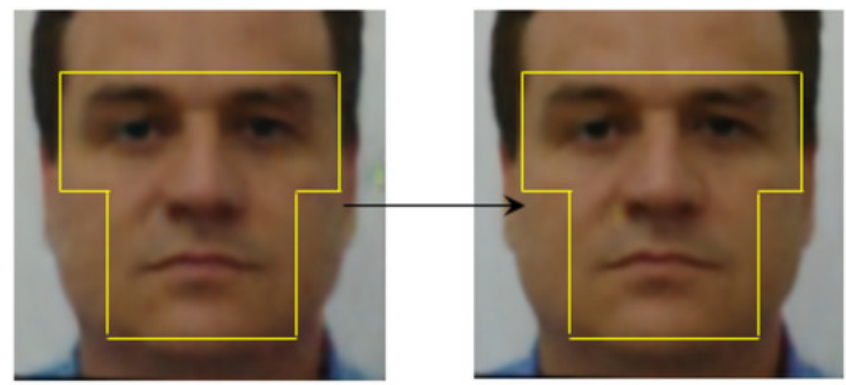

LFMTP-GAN on FEI Frontal-face TP-GAN on FEI Frontal-face $\left(90^{\circ}\right)$ $\left(90^{\circ}\right)$ 


\section{Figure 9}

A comparison of LFMTP-GAN algorithms with different techniques for face frontalization on Multi-PIE dataset.

The dataset was downloaded from the official TP-GNA GitHub page:

https://github.com/HRLTY/TP- GAN . The other image used for face recognition comparison was downloaded from: https://github.com/YuYin1/DA- GAN .
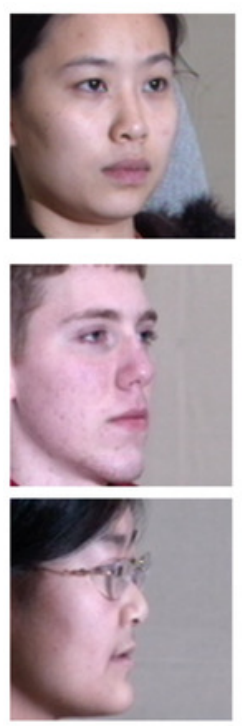

Profile image
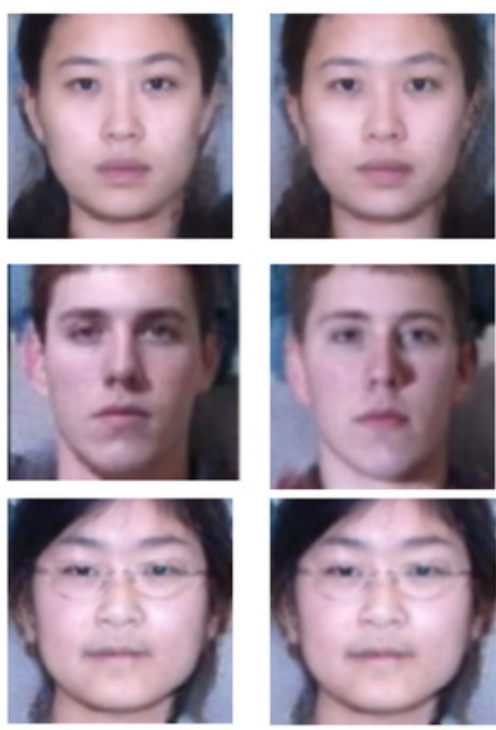

LFMTPGAN
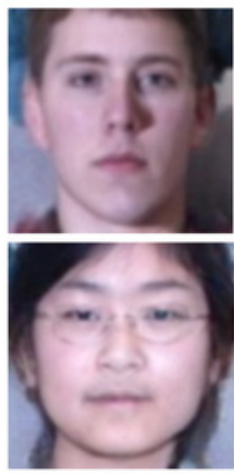

(Rui et al., 2017)

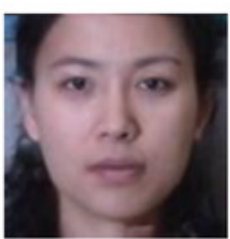

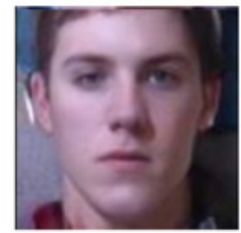

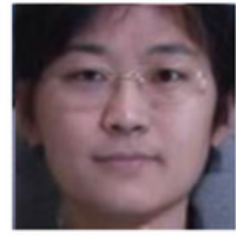

(Yu et al., 2020)
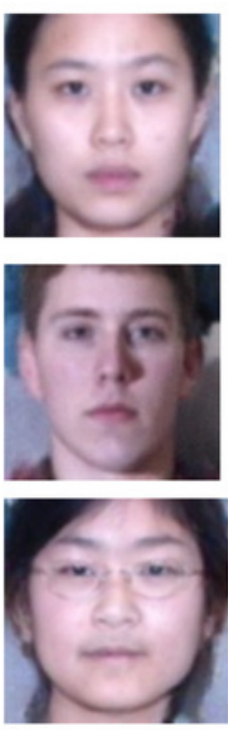

(Shuicheng \& Jiashi. 2018)
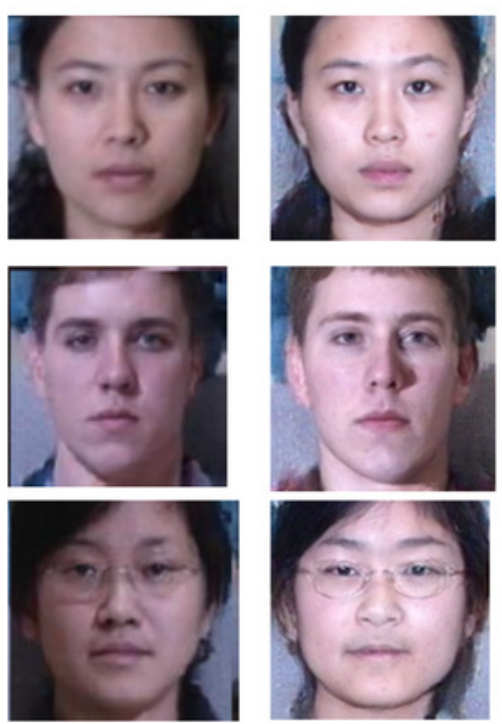

(Peipei et al,. 2019)
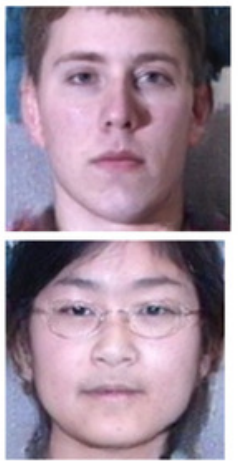

GroundTruth 


\section{Figure 10}

\section{The TP-GAN and LFMTP-GAN loss curve plots.}

Where $A$ is the generator loss curve, and $B$ is the pixel-wise loss curve. The horizontal axis indicates the number of epochs, which is the number of times that entire training data has been trained. The vertical axis indicates how well the model performed after each epoch; the lower the loss, the better a model. $C$ is the identity preserving accuracy curve, which is a quality metric that measures how accurate it is to preserve a user's identity; the higher the accuracy, the better a model. 


\section{A Generator loss}
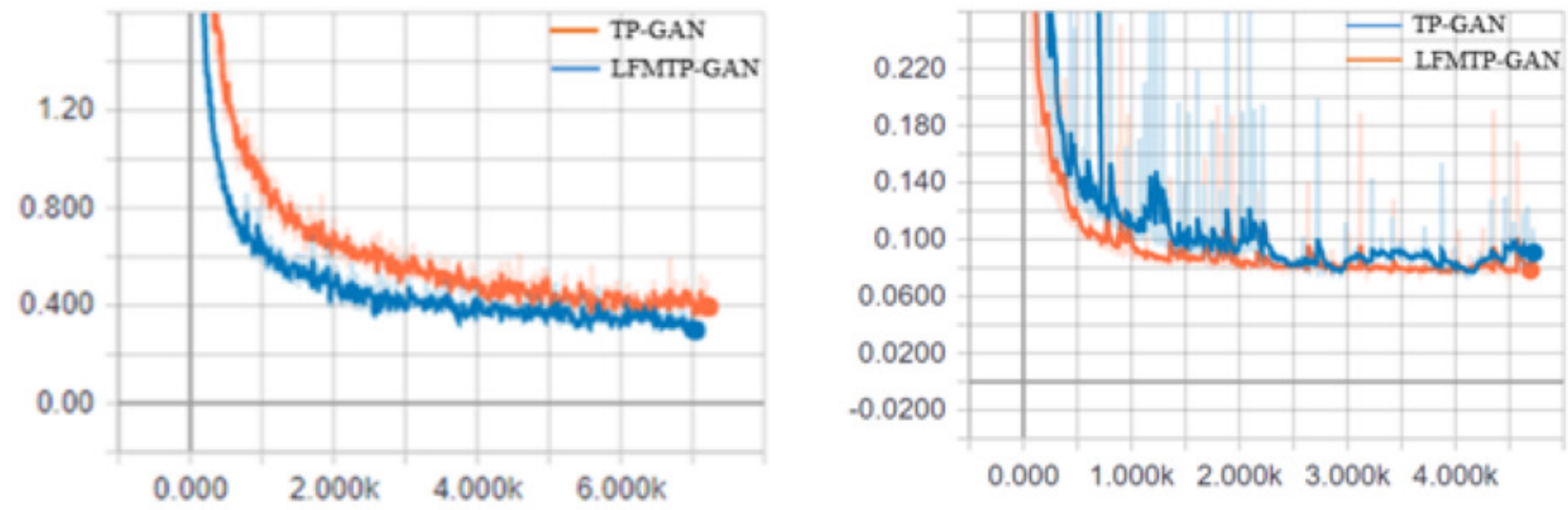

B Pixel-wise loss
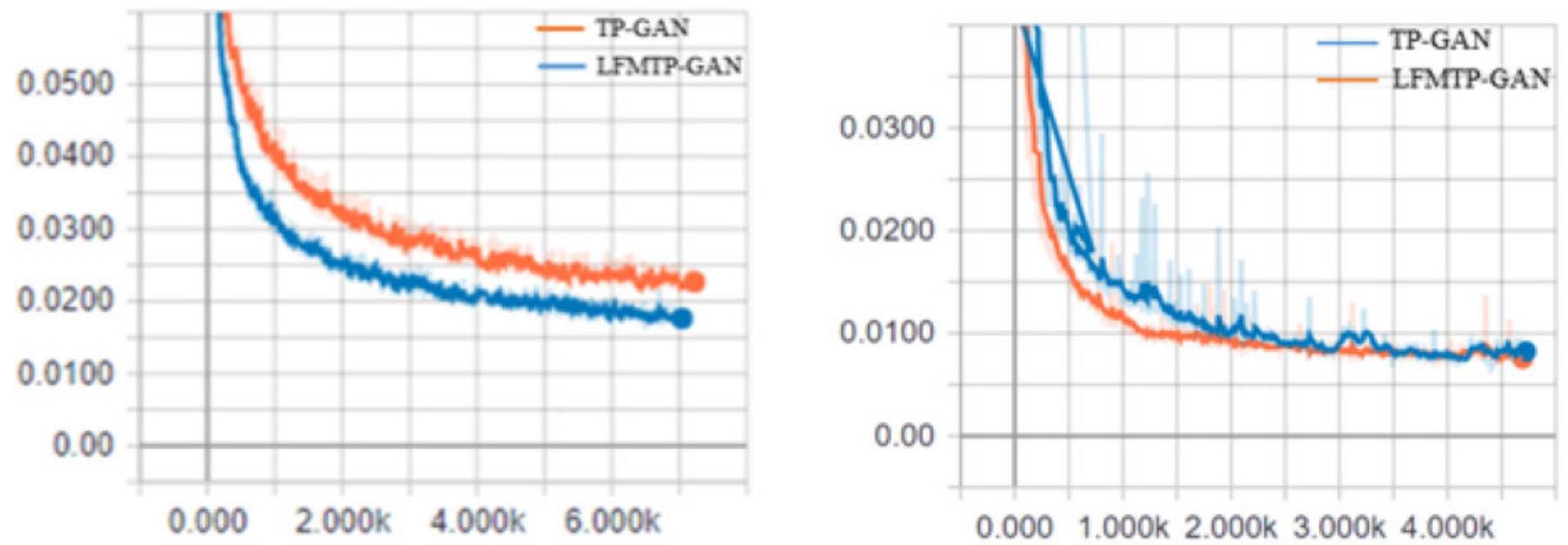

C Identity preserving accuracy
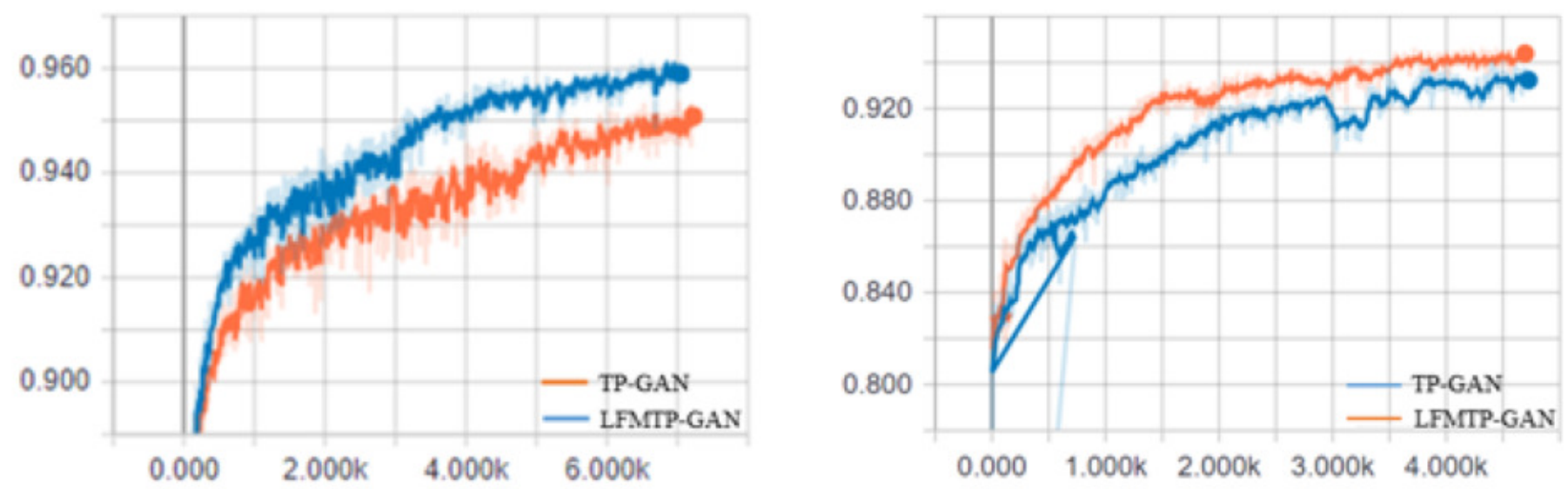


\section{Table $\mathbf{1}$ (on next page)}

An overview of the workflow of the 2D facial landmarks localization including all its operational steps. These steps are the key to our method that results in a successful implementation. 


\begin{tabular}{|c|c|}
\hline Steps & Process \\
\hline $\begin{array}{l}\text { 2D landmark } \\
\text { detection } \\
\vdots\end{array}$ & $\begin{array}{l}\text { 1- Face Detection: the goal of this step is to identify faces that are } \\
\text { generated by local and global pathways. } \\
\text { 2- Facial landmarks such as the eye centers, tip of the nose, and } \\
\text { mouth are located. } \\
\text { 3- The feature extractor encodes identity information into a high- } \\
\text { dimension descriptor. }\end{array}$ \\
\hline $\begin{array}{l}\text { Convolutional } \\
\text { neural network }\end{array}$ & $\begin{array}{l}\text { 4- The purpose of this layer is to coordinate and extract } \\
\text { intermediate features. }\end{array}$ \\
\hline $\begin{array}{l}\nabla \\
\text { Data } \\
\text { augmentation }\end{array}$ & $\begin{array}{l}\text { 5- This technique is used to enhance the synthesis image textures } \\
\text { detail by adding slightly modified copies of already existing } \\
\text { data or by creating new synthetic data based on existing data. }\end{array}$ \\
\hline 5,6 and 7 layers & $\begin{array}{l}\text { 6- } 5,6 \text { and } 7 \text { act as a visual feature map for specific inputs of } \\
\text { fontal-face images in order to retain more visual information } \\
\text { by subsampling layers' structure. }\end{array}$ \\
\hline
\end{tabular}




\section{Table 2 (on next page)}

The structure and parameters setting in the LFMTP-GAN. Those values contribute to the learning process and help our model to achieve its goals. 
1

2

\begin{tabular}{|c|c|}
\hline Parameters name & Corresponding value \\
\hline \hline Batch Size $\left(G_{\theta_{G}}\right)$ & 4 \\
\hline Batch Size $\left(D_{\theta_{D}}\right)$ & 10 \\
\hline Epoch Steps & $4500+$ \\
\hline Learning Rate $(l r)$ & 0.01 \\
\hline
\end{tabular}




\section{Table 3 (on next page)}

Cross-validation of face recognition rates on Multi-PIE and FEI datasets using weights that coordinate different loss value parameters and Table 1 settings. The run time is measured in minutes and indicates how long it will take for the model to run with 


\begin{tabular}{|c|c|c|c|c|}
\hline \multicolumn{5}{|c|}{ Multi-PIE Dataset } \\
\hline Method & Train & Valid & Cross-valid $\left(L_{s}\right)$ & Time $(\mathrm{m})$ \\
\hline TP-GAN & $95.2 \%$ & $91.7 \%$ & 0.165 & 373 \\
\hline LFMTP-GAN & $96.1 \%$ & $92.7 \%$ & 0.164 & 290 \\
\hline \multicolumn{5}{|c|}{ FEI Dataset } \\
\hline Method & Train & Valid & Cross-valid $\left(L_{s}\right)$ & Time $(\mathrm{m})$ \\
\hline TP-GAN & $94.0 \%$ & $90.0 \%$ & 0.179 & 303 \\
\hline LFMTP-GAN & $95.0 \%$ & $91.3 \%$ & 0.164 & 315 \\
\hline
\end{tabular}

1

2

3 


\section{Table 4 (on next page)}

The recognition rate (\%) across views and illuminations based on the Multi-PIE dataset under Table 1 settings. This table compares different methods of facial recognition. Our method is capable of outperforming and achieving a better result. 


\begin{tabular}{|c|c|c|c|c|c|c|c|}
\hline Mngle & $\begin{array}{c}\text { LFMTP- } \\
\text { GAN }\end{array}$ & $\begin{array}{c}\text { (Rui et al., } \\
\text { 2017) }\end{array}$ & $\begin{array}{c}\text { (Luan, Xi \& } \\
\text { Xiaoming. } \\
2017)\end{array}$ & $\begin{array}{c}\text { (Yibo et al., } \\
\text { 2018) }\end{array}$ & $\begin{array}{c}\text { (Chao et } \\
\text { al., 2015) }\end{array}$ & $\begin{array}{c}\text { (Xiang et } \\
\text { al., 2015) }\end{array}$ & $\begin{array}{c}\text { (Junho et } \\
\text { al., 2015) }\end{array}$ \\
\hline $\pm 90^{0}$ & $65.23 \%$ & $64.03 \%$ & --- & $\mathbf{6 6 . 0 5 \%}$ & $47.26 \%$ & $5.51 \%$ & -- \\
\hline $\pm 75^{0}$ & $\mathbf{8 5 . 3 0 \%}$ & $84.10 \%$ & --- & $83.05 \%$ & $60.66 \%$ & $24.18 \%$ & - \\
\hline $\pm 60^{0}$ & $\mathbf{9 4 . 1 3 \%}$ & $92.93 \%$ & $83.2 \%$ & $90.63 \%$ & $74.38 \%$ & $62.09 \%$ & - \\
\hline $\pm 45^{0}$ & $\mathbf{9 8 . 8 0} \%$ & $98.58 \%$ & $86.2 \%$ & $97.33 \%$ & $89.02 \%$ & $92.13 \%$ & $71.65 \%$ \\
\hline $\pm 30^{0}$ & $\mathbf{9 9 . 8 8 \%}$ & $99.85 \%$ & $90.1 \%$ & $99.65 \%$ & $94.05 \%$ & $97.38 \%$ & $81.05 \%$ \\
\hline $\pm 15^{0}$ & $99.80 \%$ & $99.78 \%$ & $94.0 \%$ & $\mathbf{9 9 . 8 2 \%} \%$ & $96.97 \%$ & $98.68 \%$ & $89.45 \%$ \\
\hline Average ACC & $\mathbf{9 0 . 5 2 3 \%}$ & $89.878 \%$ & $88.375 \%$ & $89.421 \%$ & $77.056 \%$ & $63.328 \%$ & $80.716 \%$ \\
\hline
\end{tabular}

